ARTICLE

\title{
ATF4 leads to glaucoma by promoting protein synthesis and ER client protein load
}

\author{
Ramesh B. Kasetti ${ }^{1}$, Pinkal D. Patel ${ }^{1}$, Prabhavathi Maddineni ${ }^{1}$, Shruti Patil (1) ${ }^{1}$, Charles Kiehlbauch ${ }^{1}$, \\ J. Cameron Millar ${ }^{1}$, Charles C. Searby ${ }^{2}$, VijayKrishna Raghunathan (iD ${ }^{3,4}$, Val C. Sheffield (1) ${ }^{2} \&$ Gulab S. Zode ${ }^{1 凶}$
}

The underlying pathological mechanisms of glaucomatous trabecular meshwork (TM) damage and elevation of intraocular pressure (IOP) are poorly understood. Here, we report that the chronic endoplasmic reticulum (ER) stress-induced ATF4-CHOP-GADD34 pathway is activated in TM of human and mouse glaucoma. Expression of ATF4 in TM promotes aberrant protein synthesis and ER client protein load, leading to TM dysfunction and cell death. These events lead to IOP elevation and glaucomatous neurodegeneration. ATF4 interacts with $\mathrm{CHOP}$ and this interaction is essential for IOP elevation. Notably, genetic depletion or pharmacological inhibition of ATF4-CHOP-GADD34 pathway prevents TM cell death and rescues mouse models of glaucoma by reducing protein synthesis and ER client protein load in TM cells. Importantly, glaucomatous TM cells exhibit significantly increased protein synthesis along with induction of ATF4-CHOP-GADD34 pathway. These studies indicate a pathological role of ATF4-CHOP-GADD34 pathway in glaucoma and provide a possible treatment for glaucoma by targeting this pathway.

\footnotetext{
${ }^{1}$ Department of Pharmacology and Neuroscience and the North Texas Eye Research Institute, University of North Texas Health Science Center at Fort Worth, TX 76107 Fort Worth, USA. ${ }^{2}$ Department of Pediatrics, Carver College of Medicine, University of lowa, lowa City, IA 52242, USA. ${ }^{3}$ Department of Basic Sciences and the Ocular Surface Institute, College of Optometry, University of Houston, Houston, TX, USA. ${ }^{4}$ Department of Biomedical Engineering, Cullen

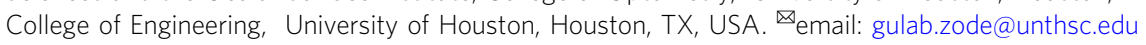


G laucoma is a heterogeneous group of optic neuropathies characterized by progressive loss of retinal ganglion cells (RGCs), degeneration of the optic nerve, and progressive visual field loss ${ }^{1-3}$. Glaucoma is the second leading cause of irreversible blindness, affecting $\sim 70$ million people worldwide 4 . In the United States, primary open-angle glaucoma (POAG) is the most common form of this disease and it is especially prevalent in African-American and Hispanic populations ${ }^{5-9}$. Elevated IOP is the most important and the only known clinically modifiable risk factor for the development and progression of POAG ${ }^{10}$. IOP is tightly regulated by aqueous humor production by the ciliary body and its outflow through trabecular meshwork (TM) tissue. In POAG, there is increased resistance to aqueous humor outflow at the TM, elevating $\mathrm{IOP}^{2,11,12}$. This increased outflow resistance is associated with several biochemical and morphological changes in the $\mathrm{TM}^{13}$. Glaucomatous $\mathrm{TM}$ damage includes increased synthesis and deposition of extracellular matrix (ECM), reduced phagocytosis, formation of cross-linked actin networks and loss of TM cellularity ${ }^{13-19}$. However, the pathological mechanisms responsible for increased outflow resistance at the TM are poorly understood.

A variety of glaucomatous insults including mutant myocilin (MYOC) and glucocorticoid (GC) therapy are known to damage $\mathrm{TM}$, elevating $\mathrm{IOP}^{20,21}$. MYOC mutations are responsible for $\sim 4 \%$ of POAG and most cases of autosomal dominant juvenileonset-open-angle glaucoma ${ }^{22,23}$. Ocular hypertension is a serious side effect of GC therapy. Approximately $30-40 \%$ of the general population develop elevated IOP as a result of topical ocular administration of potent GCs such as dexamethasone (Dex) or betamethasone. If undetected, GC-induced ocular hypertension can lead to secondary open-angle glaucoma that mimics $\mathrm{POAG}^{24-28}$. Similar to POAG, GC-induced glaucoma is caused by increased resistance to aqueous humor outflow ${ }^{21,28-30}$, as well as morphological and biochemical changes in the TM including increased ECM accumulation ${ }^{31-34}$.

Although POAG is a complex disease and caused by several factors, study of these factors has led to discovery of common pathogenic mechanisms. Recent studies have revealed the role of chronic endoplasmic reticulum (ER) stress in the pathophysiology of POAG ${ }^{35-40}$. Specifically, expression of mutant MYOC or Dex treatment leads to chronic ER stress in the $\mathrm{TM}^{37-39,41}$. The ER is a central organelle for synthesis, folding, and maturation of secretory and membrane proteins. ER stress develops when the ER is overwhelmed by unfolded and misfolded proteins, exceeding its handling capacity. In eukaryotes, ER stress is sensed by a constellation of three signal transduction pathways, collectively known as the unfolded protein response (UPR), which aid in the restoration of ER homeostasis and thus normal function ${ }^{42,43}$. The UPR can have either protective or destructive effects on cellular functions, which in turn depend on the strength and/or duration of ER stress. Chronic and sustained UPR generally causes cell death ${ }^{44,45}$. One such mechanism of chronic ER stress-mediated cell death involves activation of PERK, which leads to phosphorylation of the eukaryotic translation initiation factor (eIF2 $\alpha$ ) resulting into translational block. Activating transcription factor 4 (ATF4) is preferentially translated, which further activates downstream targets including $\mathrm{C} /$ EBP homologous protein $(\mathrm{CHOP})^{46}$. ATF4 and CHOP directly induce the growth arrest and DNA damage-inducible protein (GADD34), which further promotes dephosphorylation of eIF2 $\alpha$ leading to protein translation recovery ${ }^{47}$. There is an emerging theme that IRE1-XBP-1 activation occurs transiently in ER stress and is cell protective, whereas PERK-ATF4-CHOP activation persists during chronic ER stress and triggers cell death ${ }^{48-50}$. Although deletion of CHOP prevents ER stress-mediated cell death, recent studies have suggested an essential role for ATF4 in chronic ER stress-mediated cell death ${ }^{51,52}$. Importantly, it is not completely understood how chronic ER stress factors lead to cell death.

Although our previous studies have established that chronic ER stress is associated with TM dysfunction and IOP elevation, it is not understood whether and how chronic ER stress leads to TM dysfunction and IOP elevation. Moreover, it is not understood which signaling factors in the ER stress pathway play pathological roles in IOP elevation. Since ATF4 and CHOP are known to play pathological roles in several diseases associated with ER stress, and our recent finding that ATF4 and CHOP are significantly induced in post-mortem TM tissues from POAG donor eyes ${ }^{36}$, we further explored the roles of ATF4 and CHOP in the glaucomatous TM damage and IOP elevation.

\section{Results}

Induction of ATF4-CHOP-GADD34 pathway in human and mouse glaucoma. Our previous studies were focused on induction of CHOP in mouse models and human glaucoma donor eyes $^{36,38,39}$. In the present study, we further examined whether ATF4-CHOP-GADD34 signaling axis is also activated in TM cells and tissues of human and mouse glaucoma. We first examined markers of chronic ER stress in human primary TM cells obtained from normal and glaucoma donor eyes $(n=3-6$ different cell strains). Western blot (Fig. 1a) and its densitometric analyses (Fig. 1b) demonstrated significantly increased ATF4, CHOP, GADD34, and spliced XBP-1 protein levels in glaucomatous primary TM cells. Immunostaining for ATF4 and GADD34 in the TM tissues of human donor eyes obtained from age-matched normal $(n=9)$ and POAG patients $(n=11)$ further revealed that both ATF4 and GADD34 are significantly increased in POAG eyes (Fig. 1c, d and Supplementary Fig. 1). We next examined whether activation of ATF4-CHOP-GADD34 signaling axis is associated with IOP elevation using the recently developed mouse model of Dex-induced ocular hypertension ${ }^{53}$. C57BL/6J mice were treated weekly with periocular injections of vehicle or Dex for several weeks, and night IOPs were monitored weekly ${ }^{53}$. Dex treatment led to significant and sustained IOP elevation starting at 1-week of injection (Fig. 1e). Dex treatment led to increased IOP of $4.9 \mathrm{mmHg}$ at week $1,6.2 \mathrm{mmHg}$ at week $2,12.1 \mathrm{mmHg}$ at week $3,10.4 \mathrm{mmHg}$ at week 4 , and $8.5 \mathrm{mmHg}$ at week 5 of injections. Western blot and its densitometric analyses (Fig. 1f, g) demonstrated that GRP78, ATF4, and CHOP protein levels are significantly increased in Dex-treated iridocorneal angle tissues. Spliced XBP1 and GADD34 levels were also increased in Dextreated eyes, although this increase was not found statistically significant. In addition, immunostaining for ATF4 along with alpha smooth muscle actin (aSMA) revealed that ATF4 levels are prominently increased in the TM tissues of 5-week Dex-treated mice (Supplementary Fig. 2). Increased aSMA was also observed in the TM tissues of Dex-treated mice. Consistent with our previous studies, we observed increased ER stress (KDEL; recognizes GRP78 and GRP94) in TM of Dex-treated mice (Supplementary Fig. 3), and increased ATF4 and CHOP in GTM3 cells treated with Dex (Supplementary Fig. 4). Both ATF4 and CHOP were mostly localized to nuclei suggesting transcriptional activity. These studies indicate that ATF4-CHOP-GADD34 signaling pathway is induced in the glaucomatous TM and that induction of these proteins is associated with IOP elevation.

ATF4 elevates IOP and reduces aqueous humor outflow in mice. We next examined whether adenoviral expression of ATF4 elevates IOP in C57BL/6J mice. Three-month-old mice were injected intravitreally with Ad5.ATF4 or Ad5.Empty $\left(2 \times 10^{7} \mathrm{pfu} /\right.$ eye), and conscious IOP was measured once per week for up to 
a

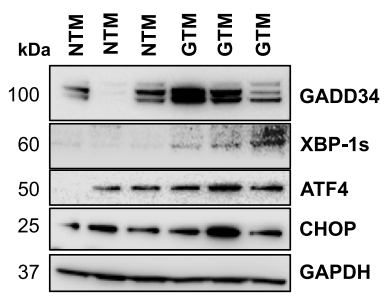

b

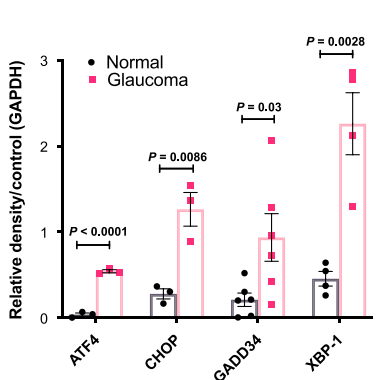

C
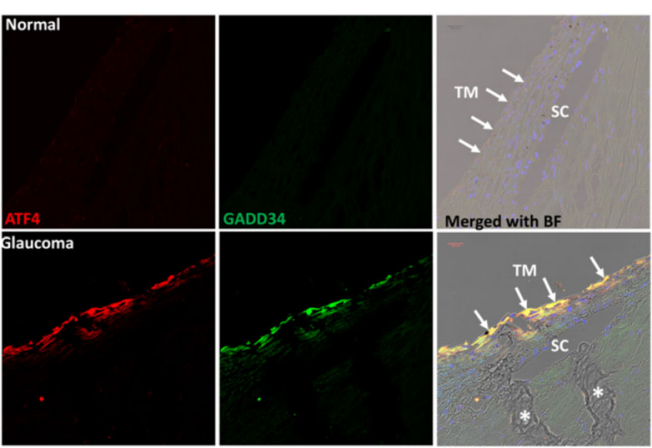

$\mathbf{9}$

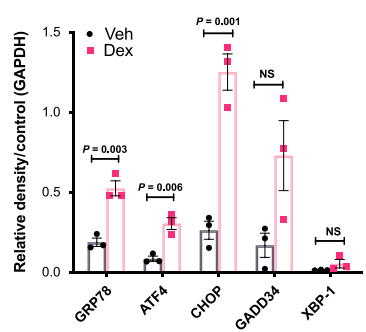

Fig. 1 ATF4-CHOP-GADD34 is induced in TM cells/tissues of human and mouse glaucoma. $\mathbf{a}$ and $\mathbf{b}$ Cellular lysates from age-matched normal and glaucomatous primary human TM cells were examined for ER stress markers using Western blotting a and analyzed by densitometric analysis $\mathbf{b}$. The average from two independent experiments is shown graphically $(n=3$ cell strains for ATF4 and CHOP; $n=6$ cell strains for GADD34; $n=4$ cell strains for XBP-1; data are presented as mean \pm SEM, 2-tailed unpaired $t$-test). $\mathbf{c}$ and $\mathbf{d}$ Representative immunostaining for ATF4 and GADD34 $\mathbf{c}$ and its intensity measurements $\mathbf{d}$ in age-matched normal and glaucoma donor eyes shows significantly increased ATF4 and GADD34 in glaucomatous TM tissues. Arrow shows TM. SC Schlemm's canal. Scale bar is $100 \mu \mathrm{m}$ ( $n=9$ normal and 11 glaucoma eyes, data are presented as mean \pm SEM, two-tailed unpaired $t$-test). *Indicates fold in section (artifact). e Periocular Dex injections elevate IOP significantly in C57BL/6J mice. 3 months old C57BL/6J mice received vehicle or Dex $(200 \mu \mathrm{g} /$ eye $)$ via periocular conjunctival fornix injections bilaterally every week up to 5 weeks and night IOPs were monitored weekly using rebound tonometry ( $n=8$ in each group; data are presented as mean \pm SEM, two-way ANOVA, ${ }^{\star \star \star} P<0.001$ ). $\mathbf{f}$ and $\mathbf{g}$ Western blot for ER stress markers $\mathbf{f}$ and its densitometric analyses $\mathbf{g}$ of anterior segment tissues from vehicle and Dex-treated mice demonstrated that induction of chronic ER stress markers is associated with Dex-induced ocular hypertension ( $n=3$ in each group, data are presented as mean \pm SEM, two-tailed unpaired $t$-test).
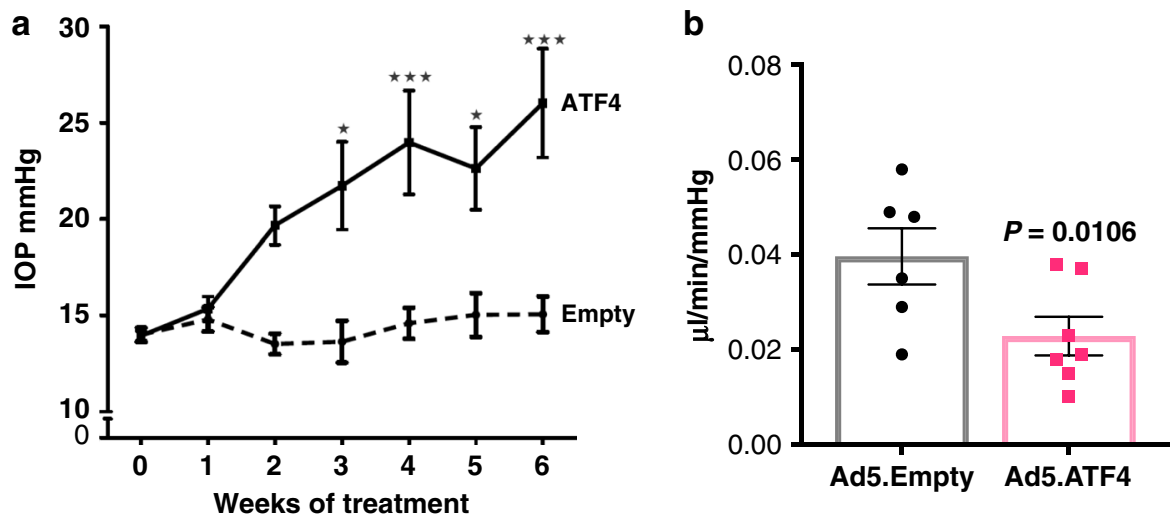

Fig. 2 ATF4 induces ocular hypertension and reduces aqueous humor outflow facility in mice. a 3 months old C57BL/6J mice received bilateral intravitreal injections of Ad5.Empty or Ad5.ATF4 ( $2 \times 10^{7}$ pfu/eye). IOPs were monitored using rebound tonometry on conscious mice. ATF4-injected mice showed significant IOP elevation starting from 3 weeks injections compared to Empty-injected mice $(n=10$ each group, data are presented as mean \pm SEM, two-way ANOVA, Bonferroni's multiple comparisons test $\left.{ }^{\star} P<0.05,{ }^{\star \star \star} P<0.001\right)$. b Conventional outflow facility was significantly reduced in Ad5.ATF4injected mice $(0.022 \mu \mathrm{L} / \mathrm{min} / \mathrm{mmHg} ; n=6)$ compared to Ad5.Empty injected mice $(0.042 \mu \mathrm{L} / \mathrm{min} / \mathrm{mmHg} ; n=6)$ (data are presented as mean \pm SEM, two-tailed unpaired $t$-test).

6 weeks (Fig. 2a). A significant and sustained IOP elevation was observed in ATF4 injected mice compared to Ad5.Empty injected mice starting at 3 weeks. We also observed that adenoviral expression of GFP did not elevate IOP significantly (Supplementary Fig. 5). We next examined whether ATF4-mediated IOP elevation is due to reduced outflow facility by measuring outflow facility using a constant flow infusion method ${ }^{54,55}$. A significant decrease in outflow facility $(0.022 \mu \mathrm{l} / \mathrm{min} / \mathrm{mmHg}$ in Ad5.ATF4 vs. $0.042 \mu \mathrm{l} / \mathrm{min} / \mathrm{mmHg}$ in Ad5.Empty; $p<0.0106)$ was observed in Ad5.ATF4 injected mice compared to Ad5.Empty injected mice 5 weeks after injection (Fig. 2b). Immunostaining of ATF4 in the anterior segment tissues revealed prominent localization of ATF4 in TM tissues of mice injected with Ad5.ATF4 while mice injected with Ad5.Empty showed little expression of ATF4 
a

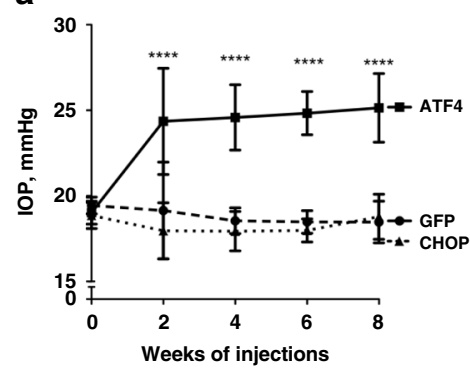

b

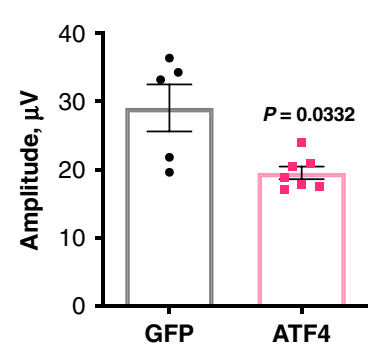

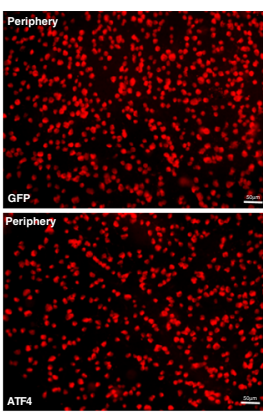
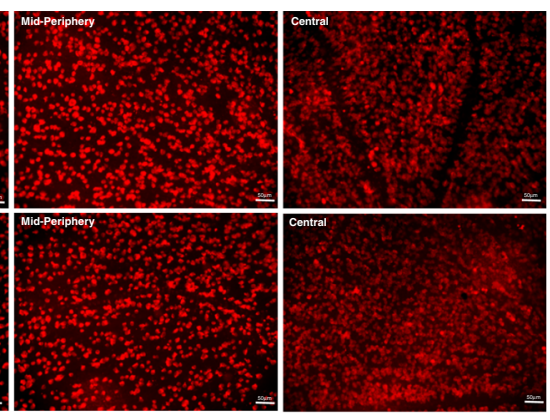

d

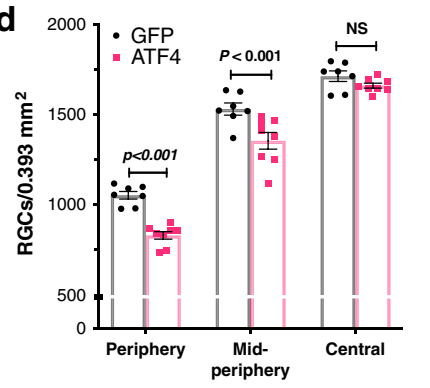

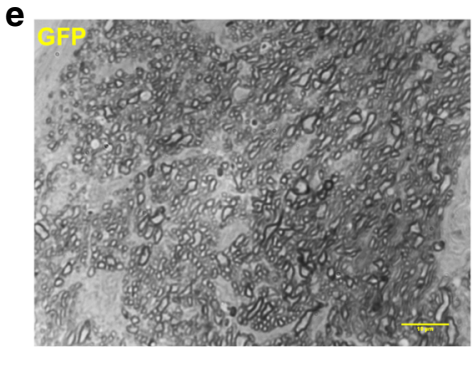

$f$

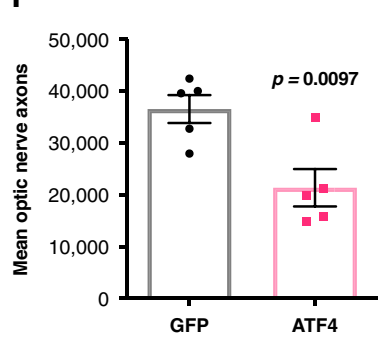

Fig. 3 Lentiviral expression of ATF4 but not CHOP induces glaucoma in mice. a Night-time IOP measurements in mice injected intravitreally with lentiviral particles expressing GFP (control), ATF4, and CHOP ( $2 \times 10^{6}$ TU/eye). A significant IOP elevation was observed in ATF4-injected mice compared to GFP or CHOP-injected mice ( $n=7$ in GFP group, $n=8$ in ATF4 and CHOP groups; data are presented as mean \pm SEM, two-way ANOVA, Tukey's multiple comparison test, $\left.{ }^{\star \star \star} P<0.0001\right)$. b RGC functional loss in ATF4-injected mice. A decrease in pattern ERG amplitudes was observed in 8 weeks ATF4-injected mice $(n=7)$ compared to GFP-injected mice ( $n=5$; data are presented as mean \pm SEM, two-tailed unpaired $t$-test. c Loss of RGCs in 10 weeks ATF4-injected mice. Representative images showing peripheral, mid-peripheral, and central regions of whole mount retinas stained with RBPMS (RGC marker) in GFP $(n=7)$ and ATF4 $(n=8)$ injected groups. d RGC counts per 0.393 mm $^{2}$ of total retina in peripheral, mid-peripheral, and central regions of ATF4 and GFP-injected mice demonstrated significant loss of RGCs in periphery and mid-periphery of ATF4-injected retinas. Scale bar is 50 m. Data are presented as mean \pm SEM, one-way ANOVA; $n=7$ for GFP and $n=8$ for ATF4. e Representative images of optic nerve sections stained with PPD showing loss of axons in 10 weeks ATF4-injected mice. Scale bar is $10 \mu \mathrm{m}$ ( $n=5$ in GFP and $n=5$ in ATF4 injected mice). f PPD stained optic nerve axons were counted and total number of axons per optic nerve were shown graphically in GFP $(n=5)$ vs. ATF4 ( $n=5)$ injected mice groups (data are presented as mean \pm SEM, two-tailed unpaired $t$-test).

(Supplementary Fig. 6). We also observed that adenoviral injections of Empty and ATF4 caused mild to moderate ocular inflammation, respectively (Supplementary Fig. 7). Although IOP elevation was sustained, examination of other glaucoma features including functional analysis of RGCs was not possible due to ocular inflammation.

Expression of ATF4 leads to glaucomatous neurodegeneration. Previous studies have shown that lentiviral particles cause minimum ocular inflammation and have selective tropism for TM cells in vivo ${ }^{56-58}$. We therefore generated lentiviral particles expressing ATF4 and CHOP and further examined whether ATF4-induced IOP elevation leads to glaucomatous neurodegeneration in mice. Two-months old C57BL/6J mice were injected intravitreally with lentiviral particles $\left(2 \times 10^{6}\right.$ TU/eye $)$ expressing GFP, ATF4, or CHOP. IOP measurements (night and day) were performed weekly (Fig. 3a and Supplementary Fig. 8). Similar to Ad5.ATF4 effects on IOP, we observed that lentiviral particles expressing ATF4 led to significant and sustained IOP elevation. Interestingly, we observed that lentiviral expression of CHOP did not elevate IOP significantly compared to mice injected with GFP. Similar results were obtained in Ad5.CHOP injected mice (data not shown) indicating that expression of $\mathrm{CHOP}$ alone is not sufficient to elevate IOP in mice. Immunostaining of ATF4 and CHOP demonstrates expression of ATF4 and CHOP in TM tissues of lentiviral injected mice (Supplementary Fig. 9). H \& E staining (Supplementary Fig. 10) reveals no ocular toxicity and open iridocorneal angles in GFP, ATF4, and CHOP injected mice.
We next examined whether ATF4 leads to functional and structural loss of RGCs, as is observed in glaucoma patients. We examined functional deficits in RGCs using pattern electroretinography $(\mathrm{PERG})^{59}$. After 8 weeks of injections, ATF4-injected mice demonstrate about $32 \%$ reduction in PERG amplitude compared to mice injected with GFP lentiviral particles (Fig. 3b). We next performed whole mount staining of retinas using the RGC-specific marker, RBPMS ${ }^{60}$. As shown in representative images, ATF4 lentiviral-injected retinas demonstrate loss of RBPMS-positive RGCs (Fig. 3c and Supplementary Fig. 11). Quantitation of RBPMS-positive cells revealed significant loss of RGCs in the periphery (21\%) and mid-periphery (12\%), but not in the central retinas of 10 -weeks ATF4-injected mice compared to the retinas of GFP-injected mice (Fig. 3c, d).

We next examined whether ATF4 leads to optic nerve degeneration by staining optic nerve cross-sections with paraphenylenediamine (PPD) and quantified optic nerve axons. As shown in representative images, optic nerve degeneration is evident in ATF4-injected mice (Fig. 3e). Quantitation of optic nerve axons demonstrated a significant loss of axons (42\%) in 10weeks ATF4-injected mice compared to GFP-injected mice (Fig. 3f). These data indicate that ATF4 leads to glaucomatous neurodegeneration in mice.

CHOP is required for ATF4-induced IOP elevation. Both ATF4 and $\mathrm{CHOP}$ are induced in glaucomatous TM tissues. However, expression of ATF4 alone but not CHOP elevates IOP significantly in mice. Since ATF4 is upstream of CHOP in the ATF4-CHOP pathway, we hypothesized that ATF4 induces 

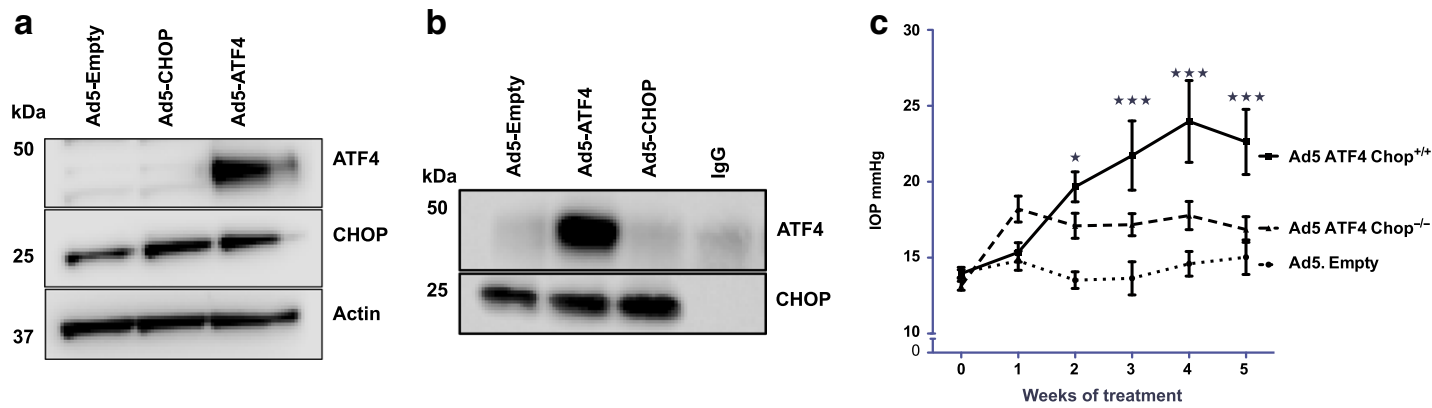

Fig. 4 CHOP is required for ATF4-induced IOP elevation. a GTM3 cells were transduced with Ad5.Empty, ATF4, and CHOP for 24 h. Cellular lysates were subjected to Western blot analysis of ATF4 and CHOP. Increased CHOP expression levels were observed in ATF4-transduced TM cells whereas CHOP transduction did not alter ATF4 levels in TM cells ( $n=3$ replicates). b GTM3 cells transduced with Ad5.Empty, ATF4, and CHOP for $24 \mathrm{~h}$. Cellular lysates were immunoprecipitated using CHOP antibody and immunoblotted for ATF4. IgG antibody was used as a negative control $(n=3$ replicates). Experiments were replicated three times independently and similar results were observed in NTM-5 cell line. c 3-month-old WT $\left(\mathrm{CHOP}^{+/+}\right)$and $\mathrm{CHOP}$ knockout $(\mathrm{CHOP}-/-)$ mice were intravitreally injected with Ad5.Empty or ATF4. A significant IOP elevation was observed in $\mathrm{CHOP}+/+$ mice injected with Ad5.ATF4 ( $n=8$ ) compared to $\mathrm{CHOP}^{+/+}$mice injected with Ad5.Empty $(n=6)$. However, ATF4 did not significantly elevate IOP in $\mathrm{CHOP}-1-$ mice $(n=8)$ compared to Ad5. Empty injected WT mice (data are presented as mean \pm SEM, two-way ANOVA with Bonferroni's multiple comparison test, ${ }^{\star} P<0.05,{ }^{\star \star \star} P<0.001$ ).

CHOP expression, and physical interaction of ATF4 with $\mathrm{CHOP}$ is required for IOP elevation. We first examined whether expression of ATF4 induces CHOP in GTM3 cells. GTM3 cells were transduced with Ad5.Empty, Ad5.ATF4, or Ad5.CHOP at $100 \mathrm{MOI}$ for $24 \mathrm{~h}$. Western blot analysis (Fig. 4a) revealed that expression of ATF4 increases CHOP expression levels, while expression of CHOP did not alter ATF4 levels in GTM3 cells. We next explored whether ATF4 interacts with CHOP in GTM3 cells by co-immunoprecipitation studies (Fig. 4b). GTM3 cells were transduced with Ad5.Empty, Ad5.CHOP, or Ad5.ATF4 for $24 \mathrm{~h}$. Total cell lysates were immunoprecipitated using anti-CHOP antibody and blotted for ATF4. Lysates incubated with IgG antibody, which showed no bands for CHOP served as a negative control. A strong interaction between CHOP and ATF4 is observed only in ATF4 transduced TM cells compared to Empty and CHOP-transduced cells. To further clarify whether the ATF4-CHOP interaction is required for IOP elevation, we performed adenoviral intravitreal injections of ATF4 in WT and Chop knockout mice. Adenoviral expression of ATF4 in Chop ${ }^{+/+}$ mice significantly elevated IOP at 2, 3, 4, and 5 weeks postinjection compared to Ad5.Empty injected Chop $\mathrm{p}^{+/+}$mice (Fig. 4c). However, expression of ATF4 did not elevate IOP significantly in $C h o p^{-1-}$ mice. These data demonstrate that $\mathrm{CHOP}$ is essential for ATF4-induced IOP elevation.

ATF4 increases protein synthesis and ER client protein load. We further explored how ATF4 leads to TM damage and IOP elevation. We first examined ER stress markers in GTM3 cells transduced with Ad5.Empty (E), ATF4, and CHOP. Western blot analysis demonstrated that expression of ATF4 but not CHOP induced spliced XBP-1 indicating that expression of ATF4 leads to induction of ER stress (Fig. 5a). Interestingly, ATF4 also induced GADD34 and decreased phosphorylation of eIF2 $\alpha$. Increased GADD34 and decreased phosphorylated eIF2 $\alpha$ suggest that ATF4 may lead to increased protein synthesis. A previous study has shown that ATF4-induced protein synthesis is associated with cell death ${ }^{51}$. We have shown that Dex increases general protein synthesis and induces secretory protein overload in TM cells ${ }^{37}$. We therefore hypothesized that induction of ATF4 leads to increased protein synthesis and ER client protein load, which may be associated with TM cell dysfunction and death. We examined de novo protein synthesis in TM cells using the SUnSET assay, a nonradioactive method of detecting de novo protein synthesis ${ }^{37}$. GTM3 cells were transfected with plasmids expressing Empty, ATF4, or CHOP for $48 \mathrm{~h}$ and incubated with puromycin for $30 \mathrm{~min}$. Total lysates were subjected to Western blot analysis using anti-puromycin antibody. ATF4 led to increased protein synthesis, which was blocked by pre-treatment with cyclohexamide (CHX) $(10 \mu \mathrm{g} / \mathrm{ml})$ (Fig. 5b). Interestingly, expression of CHOP did not alter protein synthesis compared to control cells. Since TM cells are highly secretory, we explored whether ATF4-induced protein synthesis further leads to increased ER client protein load. GTM3 cells were transfected with plasmids expressing Empty, ATF4, or CHOP for $48 \mathrm{~h}$ and incubated with puromycin for $30 \mathrm{~min}$. Total ER was isolated and equal protein from ER lysates were subjected to Western blot analysis using anti-puromycin antibody (Supplementary Fig. 12). ATF4 increased de novo protein load in the ER and also increased ER chaperones suggesting that expression of ATF4 leads to increased ER protein client load and induces ER stress in TM cells. MYOC is a secreted protein and it is highly expressed by TM cells ${ }^{61-63}$. We examined whether expression of ATF4 leads to increased accumulation of WT and mutant MYOC in TM cells. GTM3 cells stably expressing DsRed-tagged WT or mutant (Y437H) MYOC were transfected with plasmids expressing ATF4 for $48 \mathrm{~h}$ and immunostained with an ER marker, calreticulin (Fig. 5c, d), or ER stress marker, KDEL (Supplementary Fig. 13A). We observed that in control cells, WT MYOC is mostly secreted with the presence of little intracellular MYOC. Interestingly, expression of ATF4 increased WT MYOC in the ER of GTM3 cells as evident from increased co-localization with the ER marker, calreticulin (Fig. 5c). This increased intracellular accumulation of WT MYOC resulted in induction of ER stress as evident from increased KDEL staining (Supplementary Fig. 13A). KDEL antibody recognizes GRP78 and GRP94 in TM cells ${ }^{37,64}$. Furthermore, expression of ATF4 aggravated mutant MYOC accumulation and its co-localization with calreticulin in GTM3 cells stably expressing DsRed-tagged mutant MYOC (Fig. 5d). Western blot analysis of triton insoluble form of MYOC revealed that there is little or no insoluble WT MYOC in GTM3 cells expressing WT MYOC. However, expression of ATF4 increased triton insoluble WT MYOC similar to that of mutant MYOC levels (Supplementary Fig. 13B). These data further indicate that expression of ATF4 leads to increased ER client protein load in TM cells.

We next examined whether expression of ATF4 leads to ER stress in the TM in vitro and in vivo. Primary human TM cells ( $n=3$ cell strains) were transduced with Ad5.Empty, Ad5.ATF4, Ad5.ATF4 $\triangle$ RK (dominant negative inhibitor of ATF4), or Ad5. $\mathrm{CHOP}$ at $50 \mathrm{MOI}$ for 3 days. Cell lysates were subjected to 
a

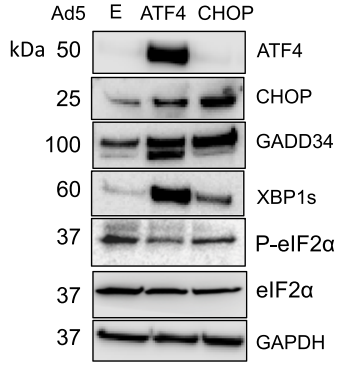

b chx

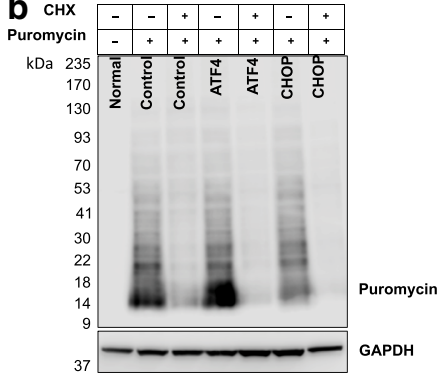

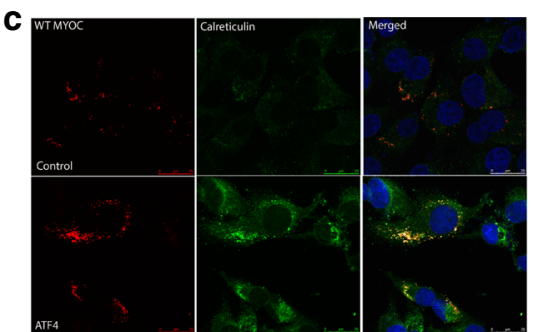

d

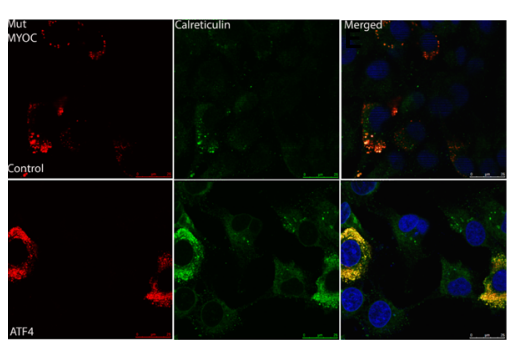

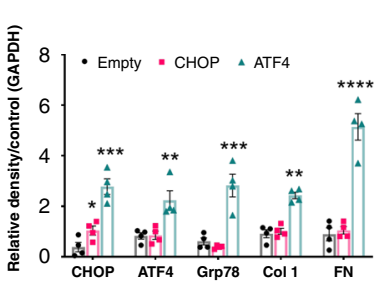

f

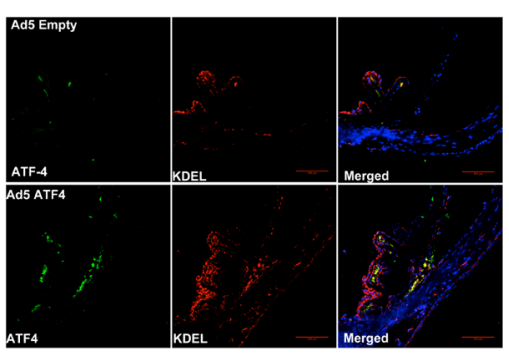

Fig. 5 ATF4 increases protein synthesis and ER client protein load in TM cells and tissues. a GTM3 cells were transduced with Ad5.Empty, ATF4, and $\mathrm{CHOP}$ for $36 \mathrm{~h}$. and cellular lysates were examined for markers of chronic ER stress by Western blot analysis. Expression of ATF4 increased XBP-1 (s) and GADD34 while ATF4 reduced p-elF2 $\alpha$ ( $n=3$ replicates). b GTM3 cells transfected with ATF4 or CHOP were incubated with cycloheximide (CHX) (10 $\mu \mathrm{g} /$ $\mathrm{ml})$ for $16 \mathrm{~h}$. Puromycin $(10 \mu \mathrm{g} / \mathrm{ml})$ was added to cells for $30 \mathrm{~min}$ before harvesting cell lysates. Total cellular lysates were subjected to Western blot analysis using anti-puromycin and GAPDH antibodies. Increased puromycin incorporation observed in the total ER fractions of ATF4 transfected cells ( $n=$ 3 replicates), which was blocked by CHX signifying a higher rate of de novo protein synthesis. $\mathbf{c}$ and $\mathbf{d}$ GTM3 cells stably expressing DsRed-tagged WT c or mutant d MYOC were transfected with plasmids expressing Empty and ATF4 for $48 \mathrm{~h}$. Cells were fixed and stained with calreticulin (ER marker). ATF4 increased intracellular WT and mutant myocilin accumulation in the ER as evident from a strong colocalization of myocilin with calreticulin in ATF4transfected cells ( $n=3$ replicates). Scale bar is $25 \mu \mathrm{m}$. e 3-month-old C57BL/6J mice were intravitreally injected with Ad5.Empty, Ad5.ATF4, and Ad5. $\mathrm{CHOP}\left(2 \times 10^{7} \mathrm{pfu} / \mathrm{eye}\right)$. After 3 weeks of injections, anterior segment lysates were subjected to Western blot (Supplementary Fig. 15) and densitometric analyses e of ECM (fibronectin, collagen-1) and ER stress (Grp78, ATF4, and CHOP) markers ( $n=4$ mice; data are presented as mean \pm SEM, one-way ANOVA, $\left.{ }^{\star} P<0.05,{ }^{\star \star} P<0.01,{ }^{\star \star \star} P<0.001,{ }^{\star \star \star \star} P<0.0001\right)$. $\mathbf{f}$ Increased ATF4 and KDEL staining in TM of Ad5.ATF4-injected mice compared to Ad5. Empty-injected mice ( $n=3$ mice). Scale bar is $100 \mu \mathrm{m}$.

Western blot analysis of ECM and ER stress markers (Supplementary Fig. 14). Adenoviral expression of ATF4 increased fibronectin, GRP78, GRP94, and CHOP compared to Ad5. Empty-treated TM cells. Transduction of TM cells with ATF4 $\triangle \mathrm{RK}$ prevented induction of fibronectin and ER stress markers. Interestingly, expression of CHOP did not alter ECM and ER stress markers. Western blot analysis further confirmed that adenoviral expression of ATF4, ATF4 $\triangle \mathrm{RK}$, and CHOP increases protein levels of ATF4 and CHOP, as expected. We also examined whether ATF4-induced ocular hypertension is associated with induction of ER stress in vivo as observed in human glaucomatous TM tissues. Three-month-old animals were administered intravitreal injections of Ad5.Empty, Ad5.ATF4, or Ad5.CHOP at $2 \times 10^{7} \mathrm{pfu} /$ eye. Western blot (Supplementary Fig. 15) and densitometric analysis (Fig. 5e) revealed that expression of ATF4, but not CHOP, significantly increases ECM markers including fibronectin and type 1 collagen (Col1). Increased CHOP and GRP78 accompanied by increased ATF4 levels were observed in the Ad5.ATF4-injected group compared to controls. Interestingly, no changes in ECM and ER stress markers were observed in the Ad5.CHOP-injected group compared to the Ad5.Empty control group. We further confirmed increased ATF4 and KDEL in mouse TM using immunostaining (Fig. 5f). ATF4 co-localized with the ER stress marker in TM tissues only in ATF4-injected mice.

ATF4 leads to TM cell death in vitro and in vivo. Since the ATF4-CHOP pathway is associated with cell death, we examined whether ATF4 leads to TM cell death in GTM3 cells. GTM3 cells were transduced with Ad5.Empty, Ad5.CHOP, or Ad5.ATF4 for $36 \mathrm{~h}$. Cellular lysates were utilized for Western blot analysis of apoptotic markers (Fig. 6a) and fixed cells were analyzed by TUNEL assay (Fig. 6b). Cleaved Caspase 3 and cleaved PARP (the classical markers of apoptosis) were observed only in Ad5.ATF4transduced GTM3 cells, but not in Ad5.CHOP-transduced GTM3 cells (Fig. 6a). Surprisingly, ATF4 did not alter death receptor 5 (DR5), which is previously shown to be involved in ER stressinduced cell death ${ }^{65}$. We observed TUNEL-positive cells only in Ad5.ATF4-transduced GTM3 cells (Fig. 6b) and in NTM5 cells (Supplementary Fig. 16). Increased numbers of TUNEL-positive cells were observed in TM tissues of Ad5.ATF4-injected mice but not in CHOP-injected mice (Fig. 6c). Together, these data demonstrate that expression of ATF4 but not $\mathrm{CHOP}$ induces apoptosis of TM cells in vitro and in vivo. Interestingly, ATF4 did not alter the expression of other pro-apoptotic markers including BAX, BAD, and DR5 suggesting that ATF4-induced TM cell death is independent of these pro-death proteins.

We next examined whether ATF4-induced apoptosis of TM cells is dependent on CHOP. GTM3 cells were transduced with Ad5.Empty, Ad5.ATF4 alone, Ad5.ATF4 plus Ad5.CRISPR targeting CHOP (CR-CHOP) or Ad5.CHOP alone. As expected, GTM3 cells transduced with ATF4 alone significantly increased cleaved PARP while knock down of CHOP significantly rescued induction of cleaved PARP compared to GTM3 cells transduced with ATF4 alone (Fig. 6d, e). These data indicate that ATF4induced TM cell death is dependent on CHOP. We next examined whether ATF4-induced protein synthesis is involved in TM cell death. Treatment with a mild dose of CHX $(2.5 \mu \mathrm{g} / \mathrm{ml})$ significantly reduced ATF4-induced cleaved PARP levels without 
a

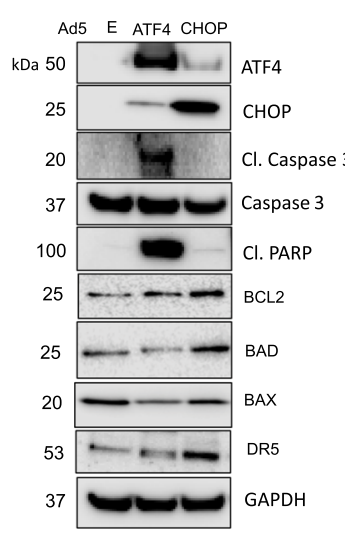

b

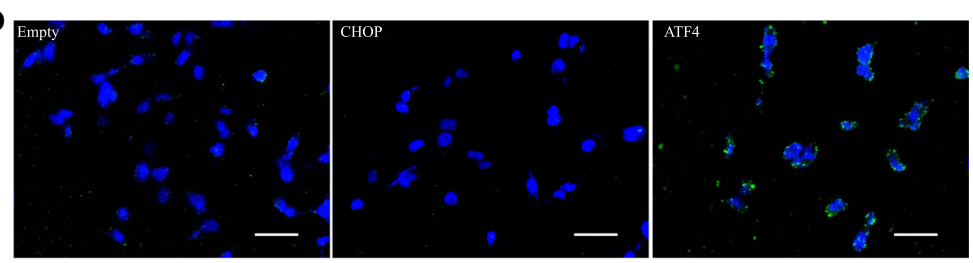

C
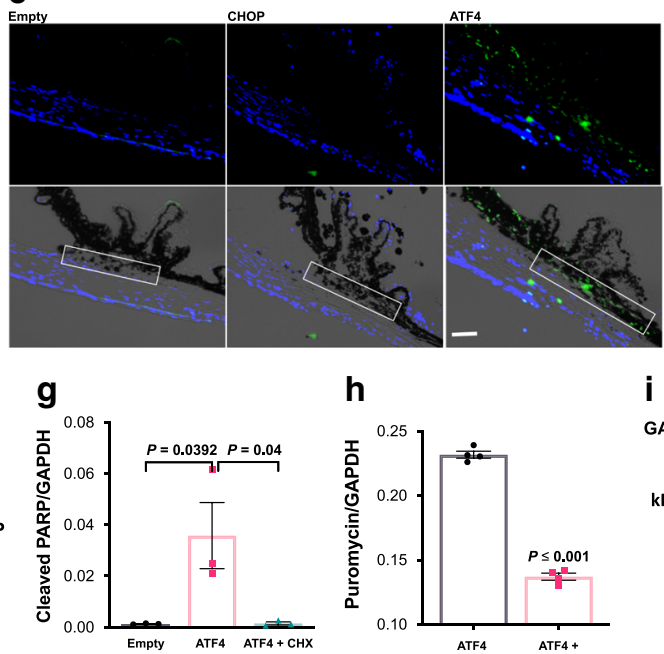

h

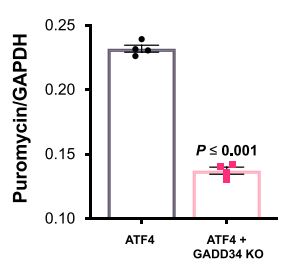

d

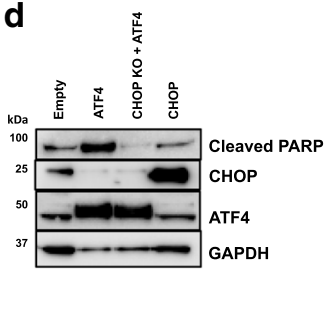

i

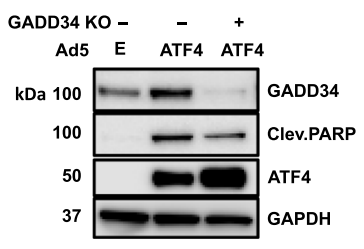

e

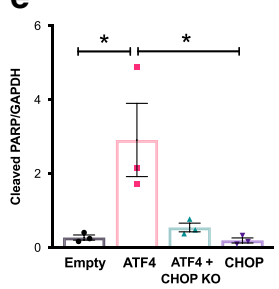

j

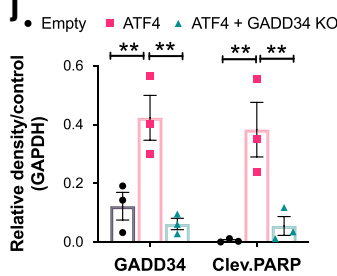

Fig. 6 ATF4 leads to TM cell death in a CHOP and GADD34-dependent manner. GTM3 cells were transduced with Ad5.Empty, ATF4, or CHOP for 36 h. a Cellular lysates were subjected to Western blot analysis for apoptotic markers ( $n=3$ independent experiments). b Fixed GTM3 cells transduced with Ad5.Empty, ATF4, or CHOP were analyzed by TUNEL assay. Increased number of TUNEL-positive cells (green) were observed in Ad5.ATF4-transduced TM cells ( $n=3$ independent experiments). Scale bar is $50 \mu \mathrm{m}$. c Increased number of TUNEL-positive cells (green) were observed in the TM region of 2 weeks Ad5.ATF4-injected mice compared to Ad5.Empty or Ad5.CHOP ( $n=3$ in each groups). Bright field images (bottom panel) merged with DAPI shows TM orientation and the TM area is represented in a white box (scale bar $=50 \mu \mathrm{m}$ ). $\mathbf{d}$ and $\mathbf{e}$ GTM3 cells were transduced with Ad5.Empty, ATF4, $\mathrm{CHOP}$, or ATF4 + CHOP KO (CRISPR-Cas9 vector targeting CHOP) for $36 \mathrm{~h}$. Western blot $\mathbf{d}$ and densitometric analyses e demonstrated that depletion of CHOP significantly reduced ATF4-induced cleaved PARP suggesting that CHOP is required for ATF4-mediated cell death. $n=3$ independent experiments, data are presented as mean \pm SEM, ${ }^{*} p<0.05$, one-way-ANOVA. $\mathbf{f}$ and $\mathbf{g}$ Western blot $\mathbf{f}$ and densitometric analyses $\mathbf{g}$ of cleaved PARP in GTM3 cells transduced with ATF4 and treated with mild dose of $\mathrm{CHX}(2.5 \mu \mathrm{g} / \mathrm{ml})$ demonstrated that reduction of protein synthesis prevents ATF4-induced TM cell death. $n=3$ independent experiments, data are presented as mean \pm SEM, one-way-ANOVA. $\mathbf{h}$ GTM3 cells expressing ATF4 were transfected with plasmid expressing CRISPR-Cas9 targeting GADD34. Puromycin $(10 \mu \mathrm{g} / \mathrm{ml})$ was added to cells for 30 min before harvesting cell lysates. Total cellular lysates were subjected to Western blot analysis using anti-puromycin and anti-GAPDH antibodies (shown in Supplementary Fig. 17). Densitometric analysis demonstrated that GADD34 knock down significantly reduces ATF4-induced protein synthesis. $n=4$ independent experiments, data are presented as mean \pm SEM, two-tailed unpaired $t$-test. $\mathbf{i}$ and $\mathbf{j}$ Western blot $\mathbf{i}$ and densitometric analyses $\mathbf{j}$ of cleaved PARP and GADD34 of GTM3 cells expressing ATF4 and plasmid expressing CRISPR-Cas9 targeting GADD34 demonstrated that knockdown of GADD34 significantly reduced ATF4induced cleaved PARP and GADD34 protein levels. $n=3$ independent experiments, data are presented as mean \pm SEM, ${ }^{\star *} p<0.01$, two-way-ANOVA.

altering ATF4 levels (Fig. 6f, g). Of note, treatment with CHX prominently reduced ATF4-induced protein synthesis (Fig. 5b). Since ATF4-induced GADD34 is known to be involved in promoting protein synthesis, we further examined whether depletion of GADD34 rescues ATF4-induced protein synthesis (Fig. 6h and Supplementary Fig. 17) and TM cell death (Fig. 6i, j). Interestingly, depletion of GADD34 prominently reduced ATF4induced protein synthesis in GTM3 cells (Fig. 6h). Furthermore, depletion of GADD34 significantly reduced ATF4-induced cleaved PARP in GTM cells (Fig. 6i, j). These studies clearly indicate that ATF4-induced TM cell death is dependent on protein synthesis and expression of CHOP and GADD34.

Several studies have linked the role of ATF4 in oxidative stress $^{66-68}$. We therefore examined whether ATF4 leads to oxidative stress in TM cells using the DCFDA assay. A significant increase in cellular reactive oxygen species (ROS) was observed upon overexpression of ATF4 in GTM3 cells (Supplementary Fig. 18A). Treatment with a chemical chaperone, sodium 4phenylbutyrate (PBA) or an antioxidant, butylated hydroxyanisole $(B H A)$ significantly reduced cellular ROS levels induced by ATF4. Since ATF4-induced cell death has been shown to be associated with oxidative stress ${ }^{51,66}$, we examined whether ATF4induced oxidative stress is a causative factor in ATF4-induced apoptosis in GTM cells. GTM3 cells transduced with Ad5.ATF4 with or without PBA or BHA (Supplementary Fig. 18B) demonstrated that depletion of oxidative stress by PBA or BHA does not rescue TM cells from ATF4-induced apoptosis.

Increased TM and SC stiffness is associated with increased outflow resistance and IOP elevation in glaucoma ${ }^{69-72}$. Cell-ECM interactions are predominant drivers of tissue stiffness. Since chronic ER stress markers were only upregulated in TM region (Fig. 1c), we further explored whether expression of ATF4 leads to altered expression of actin-regulating proteins in human TM cells and mouse TM tissues. Adenoviral expression of ATF4 increased F-actin filaments and $\alpha$-smooth muscle actin ( $\alpha$-SMA) in mouse TM tissues (Supplementary Fig. 19). Moreover, we observed that ATF4-induced expression of cleaved Rho-associated coiled coilcontaining protein kinase (ROCK)-1 and phosphorylation of myosin light chain (MLC)2 in NTM5 cells (Supplementary Fig. 20A-C). Furthermore, expression of ATF4 significantly increased phosphorylation of MLC2 in mouse anterior segment lysates (Supplementary Fig. 20D, E). These studies suggest that 
a

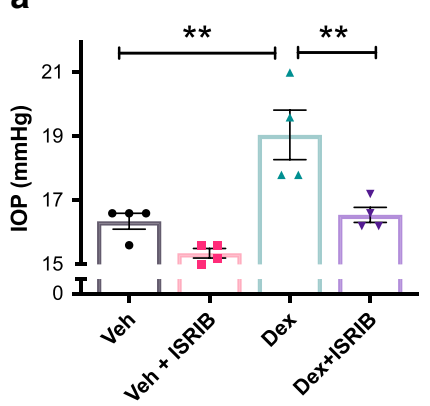

b

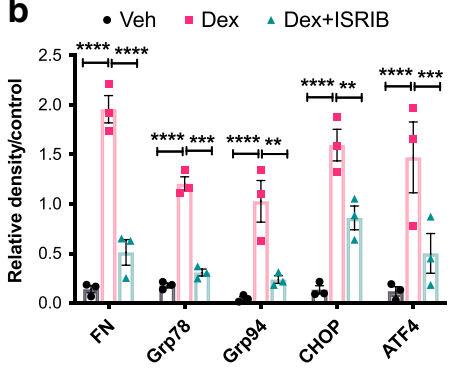

c

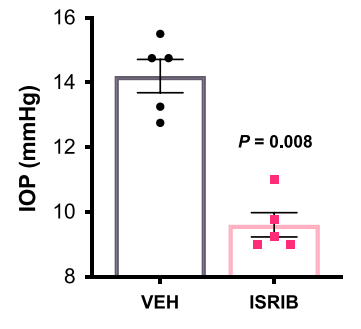

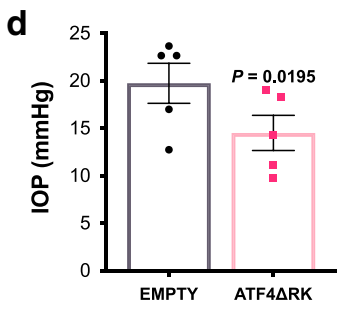

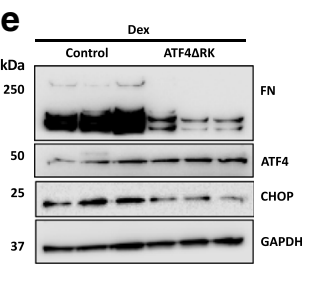

f

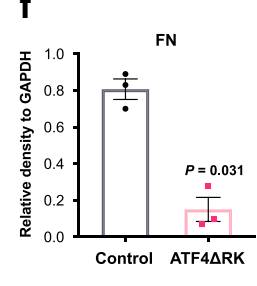

g

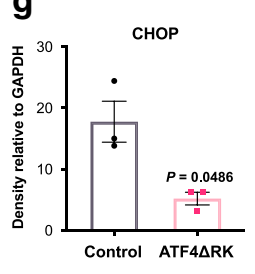

h

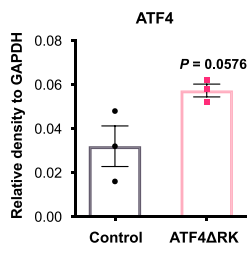

Fig. 7 Pharmacological inhibition of ATF4 rescues mouse models of glaucoma. a C57 mice were injected with vehicle $(n=4)$ and Dex $(n=4)$ weekly via periocular conjunctival fornix injections. After 2 weeks, left eyes received $5 \mu$ of $2 \mathrm{mM}$ ISRIB topical eye drops while the contralateral right eyes received vehicle eye drops (DMSO) twice daily. One-week ISRIB treatment significantly lowered IOPs in Dex-injected left eyes compared to the contralateral right eyes ( $n=4$ biologically independent samples; data are presented as mean \pm SEM, one-way ANOVA, Tukey's multiple comparison test, ${ }^{\star \star} P<0.01$ ). b Western blot (Supplementary Fig. 21) and densitometric analysis b of mouse anterior segment lysates injected with vehicle or Dex for 2 weeks and treated with $5 \mu \mathrm{l}$ of $2 \mathrm{mM}$ ISRIB topical eye drops or DMSO control. ISRIB treatment prevented Dex-induced FN and ER stress markers $(n=3$ biologically independent samples; data are presented as mean \pm SEM, one-way ANOVA, $\left.{ }^{\star \star} P<0.01,{ }^{\star \star \star} P<0.001,{ }^{\star \star \star \star} P<0.0001\right)$. c The ocular hypertensive $T g$. MYOC ${ }^{Y 437 H}$ mice received ISRIB eye drops in left eyes whereas the contralateral right eyes received vehicle (DMSO) eye drops twice daily. IOPs were recorded after one-week treatment ( $n=5$ biologically independent samples; data are presented as mean \pm SEM, two-tailed paired $t$-test). $\mathbf{d}$ C57 mice were treated with Dex for 3 weeks via periocular route. IOPs were measured after 3 weeks. After ocular hypertension was confirmed, Ad5.ATF4ARK ( $2 \times 10^{7}$ $\mathrm{pfu}$ (eye) was injected intravitreally in the left eyes while the contralateral right eyes were injected with Ad5.Empty. Both eyes were treated with Dex for another week and IOPs were measured every week. Ad5.ATF4 $\Delta$ RK reduced elevated IOP compared to contralateral eyes injected with Ad5. empty $(n=4$ biologically independent samples; data are presented as mean \pm SEM, two-tailed paired $t$-test). e-h Western blot $\mathbf{e}$ and its densitometric analyses $\mathbf{f}-\mathbf{h}$ of mouse anterior segment lysates show that ATF4 $\triangle$ RK prevented Dex-induced fibronectin and CHOP. $n=3$ biologically independent samples; data are presented as mean $\pm \mathrm{SEM}$, two-tailed unpaired $t$-test.

ATF4 modulates contractile functions of TM, which may contribute to increasing outflow resistance.

Inhibition of ATF4-CHOP pathway rescues mouse models of glaucoma. Recent studies have identified a small molecule $\mathrm{N}$, $\mathrm{N}^{\prime}$-trans-(cyclohexane-1,4-diyl)-bis-(2-(4-chlorophenoxy) acetamide (ISRIB), as a selective and potent inhibitor of the ATF4/CHOP pathway ${ }^{73,74}$. ISRIB acts downstream of elF2 $\alpha$ and upstream of the ATF4-CHOP pathway ${ }^{75}$. We examined whether inhibition of the ATF4-CHOP pathway with ISRIB reduces elevated IOP in ocular hypertensive Dex-treated or $\mathrm{Tg}-\mathrm{MYOC}^{\mathrm{Y} 437 \mathrm{H}}$ mice. C57 mice (3 months old) were treated with weekly periocular injections of vehicle or Dex, and IOPs were monitored (Fig. 7a). Within 2 weeks of treatment, Dex significantly elevated IOP compared to vehicletreated mice. At this stage, topical ocular ISRIB eye drops $(5 \mu \mathrm{l}$ of 2 $\mathrm{mM}$ stock) were given to left eyes, while the contralateral right eyes received vehicle eye drops (DMSO dissolved in PBS). IOP measurements after 1-week treatment revealed that ISRIB significantly reduces IOP in Dex-treated mice (Fig. 7a). Anterior segment tissue lysates collected from mice treated with Veh, Dex, and Dex + ISRIB were subjected to Western blot analysis of various markers of the ECM and the ER stress pathway (Supplementary Fig. 21 and Fig. 7b). Densitometric analysis confirmed that Dex significantly increases ECM and ER stress markers. ISRIB significantly reduced Dexinduced ATF4 and CHOP as well as the level of ECM and ER stress markers (Fig. 7b).
We next examined whether reduction of the ATF4-CHOP pathway reduces elevated IOP in the mouse model of MYOCassociated POAG using transgenic mice expressing mutant human MYOC ( $T g-M Y O C^{Y 437 H}$ mice). We have previously shown that $T g-M Y O C^{Y 437 H}$ mice develop ocular hypertension starting at 3 months of age and that mutant MYOC-induced ocular hypertension is associated with chronic ER stress ${ }^{38,76}$. To examine whether ISRIB reduces elevated IOP in $T g-M Y O C^{Y 437 H}$ mice, the ocular hypertensive 4-month-old $\mathrm{Tg}$-MYOC ${ }^{\mathrm{Y} 437 \mathrm{H}}$ mice were given topical ocular ISRIB eye drops $(2 \mathrm{mM})$ in the left eye, while the contralateral right eye received vehicle eye drops (Fig. 7c). IOP measurement after 1-week revealed that ISRIB significantly reduces elevated IOP in $T g-M Y O C^{Y 437 H}$ mice.

Previous studies have shown that the dominant-negative inhibitor of ATF4 (ATF4 $\triangle \mathrm{RK}$ ) inhibits transcriptional activity of endogenous $A T F 4^{66}$. We examined whether expression of ATF $4 \triangle \mathrm{RK}$ rescues Dex-induced ocular hypertension (Fig. $7 \mathrm{~d}$ ). C57BL/6J mice (3 months old) were treated with weekly periocular injections of Dex, and IOPs were monitored. After 3 weeks of Dex treatment, the left eye of Dex-treated ocular hypertensive mice were intravitreally injected with Ad5. ATF $4 \Delta$ RK while the contralateral right eyes received Ad5.Empty. IOP measurements after 1 week of treatment revealed that ATF4 $\Delta$ RK significantly reduces elevated IOP in Dex-treated mice (Fig. 7d). Western blot (Fig. 7e) and densitometric analyses (Fig. 7f-h) revealed that Ad5.ATF $4 \Delta \mathrm{RK}$ significantly reduces Dex-induced CHOP and fibronectin levels. 

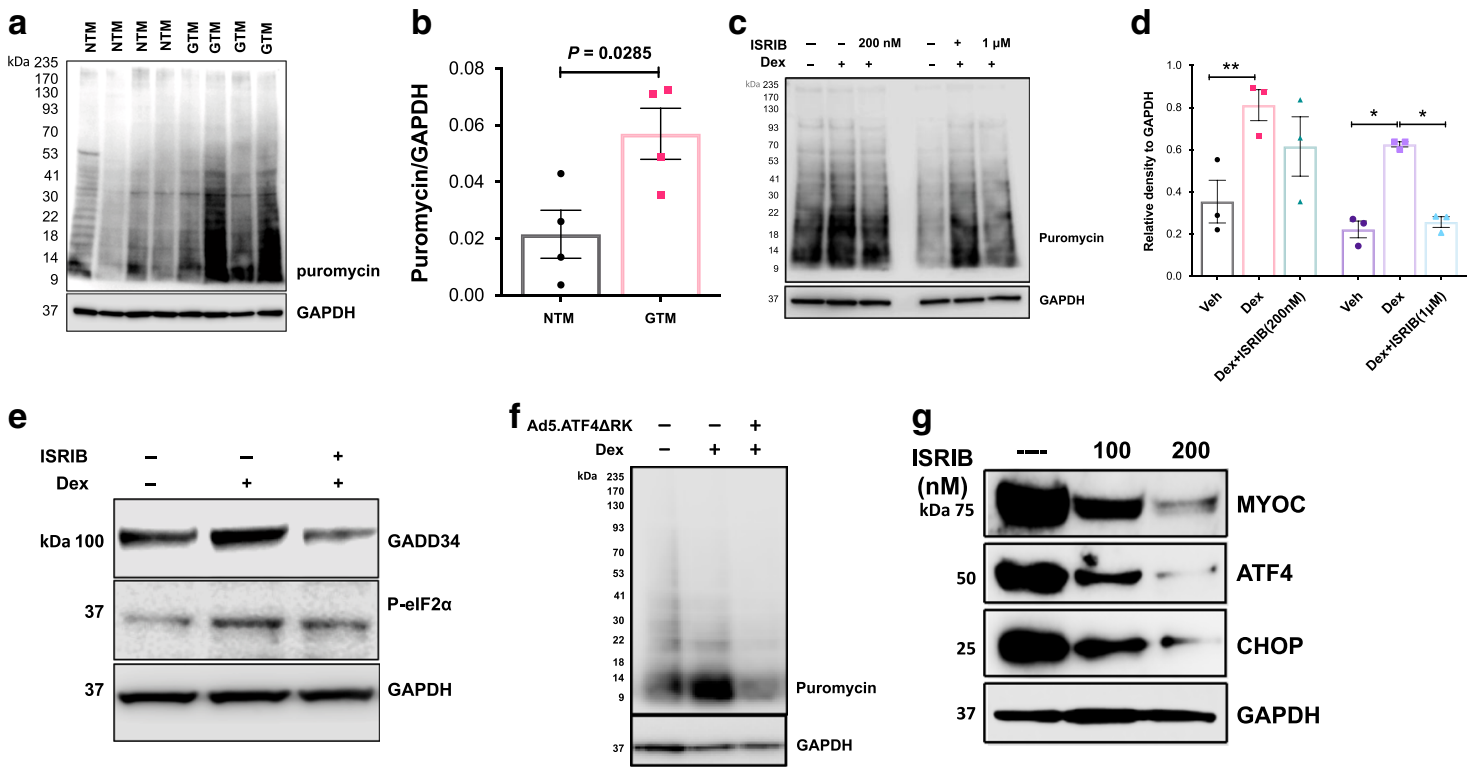

Fig. 8 Increased protein synthesis is associated glaucomatous primary human TM cells. a and $\mathbf{b}$ Protein synthesis was examined in human primary TM cells from normal ( $n=4$ biologically independent cells) and POAG donor eyes ( $n=4$ biologically independent cells) using SUnSET assay. Western blot $\mathbf{a}$ and its densitometric analysis $\mathbf{b}$ demonstrated significantly increased protein synthesis in glaucomatous TM cells. $n=4$ biologically independent samples, data are presented as mean \pm SEM, two-tailed unpaired $t$-test. c and $\mathbf{d}$ GTM3 cells were treated with vehicle and Dex along with DMSO (control) or ISRIB for $2 \mathrm{~h}$. Protein synthesis was examined using the SUnSET assay. Western blot $\mathbf{c}$ and its densitometric analysis $\mathbf{d}$ demonstrated ISRIB significantly reduced Dexinduced protein synthesis. $n=3$ independent experiments, data are presented as mean $\pm \mathrm{SEM},{ }^{\star \star} p<0.01 ;{ }^{*} p<0.05 ; 1$-way ANOVA. e Western blot analysis demonstrated that ISRIB reduced Dex-induced GADD34 levels while ISRIB effects on p-elF2 $\alpha$ were minimal. $n=3$ independent experiments. $\mathbf{f}$ Western blot analysis of total ER fractions with puromycin antibody isolated from GTM3 cells treated with vehicle, Dex, and Dex plus ATF4 $\Delta$ RK for $36 \mathrm{~h}$. Cells were incubated with puromycin antibody for 30 min before harvesting lysates ( $n=3$ independent experiments). Genetic inhibition of ATF4 prevented Dexinduced ER client protein load in GTM3 cells. $\mathbf{g}$ Western blot analysis of GTM3 cells expressing mutant myocilin treated with ISRIB showing that ISRIB reduces intracellular accumulation of mutant myocilin along with ATF4 and CHOP protein levels. $n=3$ independent experiments.

Increased protein synthesis in glaucomatous human TM cells. Since ATF4-CHOP-GADD34 are induced in glaucomatous TM cells, we further explored whether increased protein synthesis is associated with human primary TM cells obtained from POAG donors. Age-matched human primary TM cells from normal and POAG donor eyes ( $n=4$ cell strains each) were subjected to the SUnSET assay (Fig. 8a, b). Glaucomatous TM cells clearly demonstrated significantly increased protein synthesis compared to normal TM cells. We also explored whether ISRIB reduces Dex-induced protein synthesis in GTM3 cells. GTM3 cells were treated with vehicle or Dex with or without ISRIB. GTM3 cells were incubated with puromycin for $30 \mathrm{~min}$ before harvesting cell lysates. Treatment of GTM3 cells with ISRIB significantly reduced Dex-induced protein synthesis (Fig. 8c, d). Western blot analysis further revealed that ISRIB inhibits GADD34 and its effect on Dex-induced phosphorylation of eIF2 $\alpha$ is minimal (Fig. 8e). We next examined whether genetic depletion of ATF4 reduces Dexinduced ER client protein load. GTM3 cells were treated with vehicle or Dex with or without Ad5.ATF4 RR. GTM3 cells were incubated with puromycin for $30 \mathrm{~min}$ before harvesting cell lysates. ER isolates were subjected to Western blot analysis using an anti-puromycin antibody (Fig. 8f). Dex increased ER client protein load, which was reduced by Ad5.ATF4 $\Delta \mathrm{RK}$ indicating that ATF4 depletion reduces Dex-induced protein synthesis and ER client protein load in TM cells. Treatment of GTM3 cells stably expressing mutant MYOC with ISRIB reduced both ATF4 and CHOP, as well as reduced intracellular mutant MYOC accumulation (Fig. 8g) indicating that ISRIB reduces ER client protein load. These studies support that increased protein synthesis is associated with induction of chronic ER stressinduced ATF4-CHOP-GADD34 pathway and targeting this pathway rescues mouse model of glaucoma.

\section{Discussion}

Chronic ER stress is associated with TM dysfunction and IOP elevation in mouse and human glaucoma. However, it is not understood how chronic ER stress leads to TM dysfunction and IOP elevation. Here, we show that chronic ER stress-induced ATF4-CHOP-GADD34 signaling axis is activated in the TM of mouse and human glaucoma. Expression of ATF4 in TM increases de novo protein synthesis, ER client protein load and ER stress leading to TM cell death, IOP elevation and glaucomatous neurodegeneration in mice. Notably, ATF4 induces CHOP, which is required for ATF4-induced IOP elevation and TM cell death. ATF4 also induces GADD34, which is required for ATF4-induced protein synthesis and TM cell death. Genetic depletion or pharmacological inhibition of ATF4 rescues Dex or MYOC-induced IOP elevation most likely by preventing protein synthesis and reducing ER client protein load in TM cells. Importantly, increased de novo protein synthesis is associated with induction of ATF4-CHOP-GADD34 pathway in human primary TM cells from POAG donor eyes. These studies indicate a pathological role of ATF4 in glaucoma by promoting aberrant protein synthesis in TM cells.

Although the chronic ER stress pathway is implicated in several neurodegenerative diseases, its role in glaucoma is not known ${ }^{77-79}$. In the present study, we have shown that ATF4, CHOP, and GADD34 levels are increased in human primary TM cells and tissues from POAG donor eyes. In addition, we have previously shown that CHOP is increased in human glaucomatous TM tissues $^{36}$. Of note, several of glaucoma donors are likely to be treated with IOP lowering drugs, which may positively or negatively alter ER stress markers. Since similar induction of ATF4, CHOP, and GADD34 was also associated with IOP elevation in mouse model of Dex-induced ocular hypertension and in human primary TM 
cells from glaucoma donors, it is likely that induction of chronic ER stress is truly a key feature of glaucomatous TM pathology. ATF4 and CHOP can also be activated by integrated stress response (ISR) pathway ${ }^{80}$. In our models, induction of ATF4, CHOP, and GADD34 was associated with classical markers of ER stress including spliced XBP-1 and increased GRP78 and GRP94. These studies establish that chronic ER stress is the underlying key cause of ATF4-CHOP-GADD34 signaling pathway activation. To our knowledge, this is the first study that links activation of ATF4-CHOP-GADD34 signaling axis to the pathophysiology of POAG.

Forced expression of ATF4 in the TM exhibits many features of POAG including an open iridocorneal angle, reduced aqueous humor outflow and sustained IOP elevation and leads to glaucomatous neurodegeneration. In addition, expression of ATF4 increases protein synthesis and ER client protein load, leading to TM cell death. Loss of TM cellularity is observed in TM tissues of POAG donor eyes ${ }^{15}$. However, the exact cause of TM cell death is not understood. Based on our findings that ATF4 is induced in human glaucomatous TM tissues and expression of ATF4 leads to TM cell death, it is possible that induction of ATF4 in TM is associated with loss of TM in POAG. It has long been speculated that a loss of TM cells would lead to denudation of collagen structures and/or resulting in the collapse of the beams within the TM tissue. Such a collapse in the beams would decrease intertrabecular spaces thus restricting aqueous outflow and increase in IOP. Subsequently, these structural changes are likely to alter TM mechanics although this effect would be a secondary effect and not the direct causation of IOP increase. In addition to ocular hypertension, the presence of functional and structural loss of RGCs, as well as optic nerve degeneration makes this an attractive inducible mouse model to study the pathophysiology of glaucomatous TM damage and neurodegeneration.

Although adenoviral expression of ATF4 led to sustained and significant IOP elevation, it was difficult to assess glaucomatous neurodegeneration due to ocular inflammation (Supplementary Fig. 7). Ocular inflammation was observed in both Ad5.Empty and ATF4-injected mice further suggesting an immune response to adenovirus, and not the result of expression of ATF4. Previous studies have shown that Ad5 intravitreal injections can lead to mild to moderate ocular inflammation, although this inflammation does not affect IOP measurements ${ }^{81}$. Since the presence of ocular inflammation limited our ability to study RGC loss due to the presence of inflammatory cells in the retina and it is known that lentiviral particles cause minimal ocular toxicity ${ }^{58}$, we repeated these experiments using lentivirus to express ATF4. Expression of ATF4 led to sustained and significant IOP elevation without ocular inflammation. We also demonstrated that sustained IOP elevation without ocular inflammation causes glaucomatous neurodegeneration as evident from significant functional and structural RGC loss and optic nerve degeneration within 10 weeks of injection.

In our previous study, we have shown that the downstream chronic ER stress transcriptional factor, $\mathrm{CHOP}$, is also induced in the TM of POAG donor eyes ${ }^{36}$. It is known that induction of CHOP is associated with cell death and pathology in several diseases $^{82}$. It is likely that increased expression of CHOP in TM is involved in key glaucoma pathology, but expression of $\mathrm{CHOP}$ alone is not sufficient to cause these pathologies since it lacks other interacting factors of the chronic ER stress pathway including ATF4. This is supported by several observations in our study. First, ATF4 is upstream of CHOP and ATF4 induces CHOP expression, while CHOP does not induce ATF4 expression. Second, a strong interaction between ATF4 and CHOP is observed only in Ad5-ATF4-transduced TM cells but not in CHOP-transduced TM cells. Therefore, in the absence of a feedback loop, expression of CHOP does not increase ATF4, and thus sufficient interaction between ATF4 and CHOP is not possible in Ad5.CHOP-injected mice. Third, expression of ATF4 does not elevate IOP in CHOP knockout mice (in which ATF4 and CHOP interaction is obviously not possible) clearly indicating the necessity of ATF4 and CHOP physical interaction to induce IOP elevation. Our work is supported by a previous study that demonstrated that ATF4 interacts with CHOP to induce target gene expression and cell death ${ }^{51}$. The relationship between ATF4 and CHOP have been previously studied ${ }^{51,83}$, and different from the findings of our study is that CHOP alone can lead to cell death. More recent studies suggest that $\mathrm{CHOP}$ requires ATF4 to induce cell death ${ }^{51}$. Based on these studies and our findings, we propose that the effects of CHOP alone are tissue dependent. While CHOP alone may be sufficient to induce cell death in neurons, CHOP requires ATF4 to induce cell death in TM cells.

Our study points out that ATF4 promotes protein synthesis and ER client protein load, which can result in TM dysfunction and cell death leading to IOP elevation. This is evident from increased de novo protein accumulation in the ER, which is reduced after ATF4 depletion. In addition, abnormal accumulation of WT MYOC (normally secreted) in ATF4-expressing cells suggest that ATF4 leads to increased ER client protein load, which can further contribute to dysfunction of the ER leading to ER stress and cell death. Consistent with this, ATF4 leads to induction of ER stress in TM cells in vitro and in vivo. Expression of ATF4 led to increased intracellular accumulation of triton insoluble form of WT and mutant MYOC. We also observed abnormal accumulation of ECM proteins in ATF4-transduced human TM cells and mouse TM tissues. Marciniak et al. demonstrated that expression of CHOP induces cell death by increasing protein synthesis and ER protein client load ${ }^{84}$. However, we did not observe similar effects of CHOP in our experimental settings. It is thus likely that effects of CHOP alone in regulating ER client protein load are tissue specific and in the case of TM, CHOP requires ATF4 to induce such changes. Nonetheless, our study clearly demonstrates the role of ATF4 in increasing ER protein client load, which is involved in glaucomatous TM pathology. Consistent with this, we observed that both genetic and pharmacological knockdown of the ATF4 pathway rescues mouse models of glaucoma. Furthermore, ATF4 depletion reduces Dex-induced ER protein load and prevents fibrosis of the TM.

It is interesting to note that inhibition of ATF4 rescues both Dex and mutant MYOC-induced ocular hypertension. We have previously shown that CHOP is induced in $\mathrm{Tg}-\mathrm{MYOC} \mathrm{C}^{Y 437 H}$ mice and induction of CHOP is associated with TM cell death ${ }^{38}$. It is therefore possible that the ATF4-CHOP pathway is involved in aggravating MYOC misfolding and depletion of ATF4 can maintain ER homeostasis and reduce misfolded MYOC by directly inhibiting MYOC protein synthesis. Future studies will be aimed at understanding the role of ATF4 in MYOC misfolding.

It is intriguing that ISRIB reduced Dex-induced protein synthesis despite several studies have shown that ISRIB increases protein synthesis by enhancing activity of elF2B ${ }^{73,85,86}$. Since ISRIB blocks downstream effects of phosphorylated elF $2 \alpha$, it can inhibit ATF4-CHOP-GADD34 signaling and also restore general protein synthesis. We propose that ISRIB's inhibitory effect on Dex-induced protein synthesis is primarily driven by its ability to effectively block ATF4-CHOP-GADD34 signaling in TM cells. ISRIB's inhibitory effect on GADD34 would result in reduced phosphatase to inhibit phosphorylation of eIF2 $\alpha$, which will further reduce protein synthesis. In support of this, we clearly demonstrate that treatment with ISRIB significantly reduces Dexinduced protein synthesis in TM cells, which is associated with a strong reduction in GADD34, ATF4, and CHOP levels while its 
effects on phosphorylation of eIF $2 \alpha$ are minimal. A recent study by Wang et al. (2019) independently demonstrated that the elF2 $\alpha$ dephosphorylation inhibitor, Salubrinal prevents tunicamycininduced TM cell death ${ }^{87}$. Moreover, dominant negative inhibitor of ATF4 reduced Dex-induced protein synthesis and ocular hypertension. Alternatively, there may be another pathway by which GADD34 directly regulates protein synthesis. It is also likely that ISRIB has differential effects on chronic ER stress compared to ISR since most of effects of ISRIB are studied in context of the ISR pathway. In line with this, another study by Rabouw et al. (2019) demonstrated that ISRIB promotes protein synthesis when p-eIF2 $\alpha$ levels are low but ISRIB is ineffective when p-eIF2 $\alpha$ levels are high (in context of ISR) ${ }^{74}$

In summary, our studies indicate that ATF4-CHOP-GADD34 signaling pathway is induced in glaucoma, which promotes protein synthesis and ER client protein load, inducing TM cell death and IOP elevation. ATF4CHOP-GADD34 signaling axis plays a pathological role in TM dysfunction leading to IOP elevation and inhibition of this pathway represents an attractive therapeutic target for slowing the progression of this disease.

\section{Methods}

Antibodies and reagents. Antibodies were purchased from the following sources; fibronectin (catalog \# Ab2413, Abcam), KDEL (catalog \# Ab12223, Abcam), collagen I (catalog \# NB600-408, Novus Biologicals), ATF4 (catalog \# SC-200, Santa Cruz Biotechnology), CHOP (catalog \# 13172, Novus Biologicals), GRP78 (catalog \# ab21685, Abcam), GRP94 (catalog \# 11402, Santa Cruz Biotechnology), Caspase 3 (catalog \# 9662, Cell signaling technology), Cleaved PARP (catalog \# 9541, Cell signaling technology), puromycin (catalog \#A11138, Gibco, Life Technology), RBPMS (catalog \# 118619, Gene Tex), cleaved caspase 3 (catalog \# 9661, Cell signaling technology), GAPDH (catalog \# 3683, Cell signaling technology), and $\beta$ Actin (catalog \# 4970, Cell signaling technology, Danvers, MA, USA). For immunoprecipitation studies, a different CHOP (sc-7351, Santa Cruz Biotechnology) was used. All adenoviral vectors used in this study were obtained from ViraQuest Inc. (North Liberty, IA, USA). Lentiviral vectors expressing ATF4 or CHOP were obtained from VectorBuilder Inc.

Experimental animals. 2 to 3 -month-old C57BL/6J mice were utilized in this study. Animals were purchased from the Jackson Laboratory (Bar Harbor, ME, USA). Animals were allowed to roam freely in their cages and had access to food (standard mouse chow) and water ad libitum and were maintained under $12 \mathrm{~h}$ light/12dark conditions. All experimental procedures were conducted in accordance with and adherence to the ARVO Statement for the Use of Animals in Ophthalmic and Vision Research. Experimental protocol was approved by the Institutional Animal Care and Use Committee (IACUC) of the University of North Texas Health Science Center (UNTHSC) (Protocol \#: IACUC-2018-0032). Chop knock out mice were obtained from the Jackson labs.

Viral vector injections. Adenovirus expressing empty, GFP, CHOP, ATF4WT, and ATF4 $\triangle \mathrm{RK}$ under the control of CMV promoter were purchased from ViraQuest $^{66}$. Lentiviral particles expressing GFP, CHOP, and ATF4 were purchased from VectorBuilder. Intravitreal injections of viral particles were performed ${ }^{41}$. Anaesthetized mice were injected with Ad5 expressing either empty or CHOP or ATF4 $\left(2 \times 10^{7} \mathrm{pfu} / \mathrm{eye}\right)$ in vitreous chamber through sclera using a Hamilton syringe fitted with a sterile 33-gauge needle. Similar to Ad5, high titer ultra-pure lentiviral particles expressing either GFP or ATF4 WT or CHOP $\left(2 \times 10^{6}\right.$ TU/eye $)$ under the control of $\mathrm{CBh}$ promoter were injected intravitreally in both eyes.

Cell culture. Age-matched primary human TM cells (between passages 5 and 7) from normal and POAG donor eyes (four strains each) and transformed GTM3 or NTM5 cells were cultured in DMEM supplemented with 10\% FBS (Hyclone Laboratories), $2 \mathrm{mM}$ L-glutamine, penicillin (10,000 units $/ \mathrm{ml})$, and streptomycin $(10 \mu \mathrm{g} / \mathrm{ml})($ Gibco BRL). Cells were maintained in a humidified incubator at $5 \%$ $\mathrm{CO}_{2}$ and $37^{\circ} \mathrm{C}$. Adenoviral transductions were performed using antibiotic-free medium at 100 MOI for $24 \mathrm{~h}$. Cells were then transferred to fresh medium and maintained for another $48 \mathrm{~h}$. Cell lysates were collected in $1 \times$ lysis buffer containing: HEPES $(50 \mathrm{mM}), \mathrm{KCl}(200 \mathrm{mM})$, EDTA $(2 \mathrm{mM}), \mathrm{MgCl}_{2}(1 \mathrm{mM})$, Triton X-100 (0.5\%), glycerol (10\%), $0.5 \mathrm{mM}$ dithiothreitol, and protease inhibitor cocktail tablets (Roche Life Sciences, Indianapolis, IN, USA). For Dex treatment, cells were transduced with appropriate viral vectors for $24 \mathrm{~h}$ and treated with vehicle or Dex $(100 \mathrm{nM})$ for another 3 days $^{88}$. GTM3 cells stably expressing DsRed-tagged WT and mutant $(\mathrm{Y} 437 \mathrm{H})$ MYOC were generated previously ${ }^{76}$. These cells were treated with ISRIB or plasmids expressing Empty or ATF4 to examine effects on MYOC intracellular accumulation. To examine whether CHX inhibits ATF4-induced protein synthesis, GTM3 cells transduced with ATF4 or CHOP were incubated with $\mathrm{CHX}(10 \mu \mathrm{g} / \mathrm{ml})$ for $16 \mathrm{~h}$ and cell lysates were subjected to further analysis. To examine whether reduction of ATF4-induced protein synthesis prevents TM cell death, GTM3 cells transduced with ATF4 were incubated with CHX $(2.5 \mu \mathrm{g} / \mathrm{ml})$ for $12 \mathrm{~h}$ before harvesting cellular lysates. CRISPR-Cas9 targeting CHOP or GADD34 were generated ${ }^{41}$. GTM3 cells were first transfected with plasmids expressing CRISPR-Cas 9 targeting CHOP or GADD34 for $24 \mathrm{~h}$ and then transduced with plasmids or adenovirus expressing ATF4 for $36 \mathrm{~h}$.

Intraocular pressure (IOP) measurement. IOP measurements were carried out using TonoLab ${ }^{\circledast}$ rebound tonometer (Colonial Medical Supply, Franconia, NH, USA $)^{39,89}$. All IOPs were measured during the day between 10 a.m. and 2 p.m. in a masked manner. Six individual IOP measurements were averaged to obtain the final IOP value for each eye at each time point. For measuring night-time IOPs, mice were kept in dark for $7 \mathrm{~h}$ (3-10 p.m.) and IOPs were measured in the dark using dim red lights ${ }^{38}$.

Aqueous outflow facility (C). Aqueous humor outflow facility $(C)$ was established by constant flow infusion following our previously published methodology ${ }^{54,55}$. In brief, animals were anesthetized by intraperitoneal (i.p.) injection of a cocktail of ketamine $(10 \mathrm{mg} / \mathrm{ml})$ and xylazine $(1 \mathrm{mg} / \mathrm{ml})$ given in a volume of $10 \mathrm{ml} / \mathrm{kg}$ for induction. Both eyes then received a drop of proparacaine $\mathrm{HCl}(0.5 \%)$ for topical anesthesia. The anterior chamber was cannulated with a $30 \mathrm{G}$ steel needle connected to a previously calibrated (sphygmomanometer, Diagnostix 700, Hauppage, NY, USA) BLPR-2 flow-through blood pressure transducer (World Precision Instruments (WPI), Sarasota, FL, USA) for the continuous determination of pressure within the eye. A topical ocular drop of sterile phosphate buffered saline (PBS, Amresco ${ }^{\circ}$, Solon, OH, USA) was also applied to each eye to prevent corneal drying. The opposing end of each transducer was connected to a three-way valve, which in turn was connected to: (1) $50 \mu$ l glass microsyringe (Hamilton Company, Reno, NV, USA) filled with sterile PBS (previously filtered through a $0.2 \mu \mathrm{m}$ HT Tuffryn Membrane Acrodisc syringe filter; PALL Gelman Laboratory, Port Washington, NY, USA) loaded into SP101i microdialysis infusion pump with halfstepping capability (WPI), and (2) an open-ended, variable-height manometer. Signals from the pressure transducers were passed via a TBM4M Bridge Amplifier (gain setting: $1000 \times$, WPI) and a Lab-Trax analog-to-digital converter (WPI) to a computer. Data were recorded using Lab-Scribe2 software (WPI).

The manometer was opened to the circuit and the pressure adjusted to equal the pre-cannulation IOP. Following a period of $\sim 1 \mathrm{~min}$, the manometer was closed to the circuit and eyes were infused at a flow rate of $100 \mathrm{~nL} / \mathrm{min}$. When pressure had stabilized (typically within 10-30 min), pressure measurements were recorded every $5 \mathrm{~min}$ for the next $15 \mathrm{~min}$. Flow rates were then increased sequentially (200, 300,400 , and $500 \mathrm{~nL} / \mathrm{min}$ ). Three stabilized pressures (spaced $5 \mathrm{~min}$ apart) were recorded at each flow rate. $C$ in each eye of each animal was calculated as the reciprocal of a slope of a plot of mean stabilized pressure as ordinate against flow rate as abscissa.

Mouse slit-lamp examination. Cornea and anterior chamber phenotypes were examined with a slit-lamp (SL-D7; Topcon) and photo-documented with a digital camera (D100; Nikon) ${ }^{38}$.

Pattern ERG. RGC function was analyzed using binocular snout pattern ERG (PERG animal research system, JORVEC Corp., Miami, FL, USA) ${ }^{59}$. In brief, mice were anesthetized using intraperitoneal injection of ketamine/xylazine mixture $(100,10 \mathrm{mg} / \mathrm{kg}$, respectively). Anesthetized mice were placed on a temperature controllable metal base at a fixed distance $(10 \mathrm{~cm})$ from the LED monitors and maintained at constant body temperature $\left(37^{\circ} \mathrm{C}\right)$ using a rectal probe. A small amount of hypromellose eye drops were applied topically to prevent corneal dryness while recording. The PERG was derived simultaneously from each eye using subcutaneous electrodes placed at snout (active), back of the head (reference) and tail (ground) in response to contrast reversal of gratings generated from two LED screens, operated at a slightly different frequency. Two consecutive repetitions were averaged and amplitudes (P1-N2) representing RGC function was graphically shown.

RGC staining and counting. Mice were euthanized, and enucleated eyes were fixed in $4 \%$ PFA overnight at $4^{\circ} \mathrm{C}$. Next day, eyes were washed thoroughly with 1xPBS and dissected into anterior and posterior cups. Whole retina was removed from the posterior cups, incubated in blocking buffer ( $10 \%$ goat serum containing $1 \%$ Triton-X-100) for $3 \mathrm{~h}$. Retina was then incubated with primary antibody (Rabbit antiRBPMS, 1:200) in a blocking buffer for $48 \mathrm{~h}$ at $4{ }^{\circ} \mathrm{C}$. Retina was washed thrice with $1 \times$ PBS containing $0.1 \%$ Triton-X-100 for 30 min each. Retina was incubated with appropriate secondary antibody (Alexa flour anti-rabbit 568, 1:500) in $1 \times \mathrm{PBS}$ for $2 \mathrm{~h}$. Retina was washed thrice with $1 \times \mathrm{PBS}$ for $30 \mathrm{~min}$ each and flat-mounted on a glass slide by cutting four equal quadrants attached at center of the retina. Images were taken using Keyence microscope at $\times 200$ magnification. Total of 20 nonoverlapping images per the retina including 12 from periphery ( 4 from each quadrant), 4 from mid-periphery ( 1 from each quadrant), and 4 from centra 
region (1 from each quadrant) were taken and used for counting of RBPMSpositive RGCs using Image J software. The total number of RGCs in peripheral, mid-peripheral, and central retina were quantified separately in each group and represented graphically.

PPD staining of optic nerve. Axonal degeneration in the optic nerve was examined using a PPD staining and total mean optic nerve axons were counted ${ }^{38}$. Briefly, optic nerves were collected from GFP and ATF4-injected mice and fixed overnight in phosphate buffer solution containing 3\% glutaraldehyde/paraformaldehyde at $4^{\circ} \mathrm{C}$. The optic nerves rinsed twice for $10 \mathrm{~min}$ with $0.1 \mathrm{M}$ phosphate buffer and once with $0.1 \mathrm{M}$ sodium acetate buffer, then dehydrated in graded ethanol concentrations. After embedding in the resin, the transverse semithin sections $(1 \mu \mathrm{m})$ were cut and stained with $1 \%$ PPD for $10 \mathrm{~min}$. Total 10 images without overlap were taken using brightfield microscope (Leica DM 2000 LED) with a total amplification of $\times 1000$. The surviving axons were counted in area of 625 square micrometers from each image and sum of total surviving axons counted approximately equals to $10 \%$ of the total optic nerve cross-sectional area.

TUNEL assay. TUNEL assays were performed on TM cells and mouse eye sections using a TUNEL Apo-Green Detection Kit (Biotool, Houston, USA) in accordance with the manufacturer's recommended protocol. Two weeks after Ad5.Empty or Ad5.ATF4 injections $\left(2 \times 10^{7} \mathrm{pfu} / \mathrm{eye}\right)$, animals were sacrificed and eyes were enucleated, fixed, processed, and embedded in paraffin. Five-micron sections were cut using a microtome. Sections were dewaxed, rehydrated, and permeabilized by incubating with $20 \mu \mathrm{g} / \mathrm{ml}$ proteinase $\mathrm{K}$ solution for $20 \mathrm{~min}$ at room temperature. GTM3 or NTM5 cells transduced with Ad5.Empty or Ad5.ATF4 (100 MOI) for $36 \mathrm{~h}$ and were fixed in freshly prepared 4\% PFA and permeabilized using $0.2 \%$ triton-X-100 for $10 \mathrm{~min}$. Both mouse eye sections and fixed TM cells were incubated with $1 \times$ equilibration buffer for $10 \mathrm{~min}$ and allowed $60 \mathrm{~min}$ incubation with the TUNEL reaction mixture (containing Apogreen labeling mix and Recombinant TdT enzyme) at $37^{\circ} \mathrm{C}$ in the dark. Following three washes in PBS, sections were mounted in DAPI-mounting solution. TUNEL-positive green fluorescence images were taken using a Keyence microscope (Itasca, IL, USA).

Immunostaining. Enucleated eyes were fixed in $4 \%$ paraformaldehyde for $3 \mathrm{~h}$. The fixed eyes were processed and embedded in paraffin. Five-micron sections were cut and utilized for immunostaining. Briefly, sections were deparaffinized and rehydrated, then subjected to antigen retrieval with citrate buffer $(\mathrm{pH}=6)$. Slides were incubated with blocking buffer (10\% goat serum and $0.5 \%$ Triton-X-100 in PBS) for $2 \mathrm{~h}$, and then with primary antibody in blocking buffer overnight. Slides were washed three times with PBS, and then incubated for $2 \mathrm{~h}$ with approximate Alexa Fluor secondary antibodies (Life technologies, Grand Island, NY, USA). After three final washes in PBS, sections were mounted with DAPI-mounting solution. Images were captured using a Keyence microscope (Itasca, IL, USA). For immunostaining of human donor eyes, age-matched normal $(n=9)$ and glaucomatous $(n=11)$ anterior segment sections were stained with ATF4 and GADD $34^{90}$. The study design and conduct complied with all relevant regulations regarding the use of human study participants and was conducted in accordance to the criteria set by the Declaration of Helsinki. Briefly, post-mortem fixed human eyes were received from the Lions Eye Institute for Transplant \& Research (Tampa, FL). The Lions Eye Institute is regulated directly by the Food and Drug Administration (FDA) and it is exempted from oversight of an Institutional Review Board (IRB), since it does not conduct biomedical research involving living human research subjects. These tissues are self-donated to the Lions Eye Institute for research and Lions Eye Institute receives informed consent and complies with the Florida Agency for Healthcare Administration (AHCA) statute 59A-1 before collecting tissues.

Co-immunoprecipitation. GTM3 cells were transduced with Ad5-Empty, Ad5CHOP, and Ad5-ATF4 at 100 MOI for $24 \mathrm{~h}$. Co-immunoprecipitation was carried out with anti-CHOP antibody using the Dynabeads Co-Immunoprecipitation Kit (Life technologies, Grand Island, NY, USA). Briefly, CHOP and IgG antibodies (2 $\mu \mathrm{g}$ ) were diluted in $200 \mu \mathrm{l}$ of lysis buffer (used as binding and washing buffer) and incubated with $50 \mu \mathrm{l}$ of dynabeads protein $\mathrm{G}$ for $10 \mathrm{~min}$ at room temperature on a rotating shaker. Bead- $\mathrm{Ab}$ complexes were washed three times using the magnet separator provided with the kit. Total protein lysates $(200 \mu \mathrm{g})$ were incubated with bead-Ab complexes for $10 \mathrm{~min}$. Beads- $\mathrm{Ab}-\mathrm{Ag}$ complexes were washed three times with lysis buffer containing $0.5 \%$ Triton-X-100. The bead-Ab-Ag complexes were then dissolved in $30 \mu \mathrm{l}$ of LDS sample buffer and heated for $10 \mathrm{~min}$ at $70^{\circ} \mathrm{C}$. Beads were separated using the magnet separator, and supernatants were subjected to Western blot analysis.

Western blot analysis. Approximately $20-30 \mu \mathrm{g}$ total protein from either cell lysates or anterior segment lysates were run on denaturing $4-12 \%$ gradient polyacrylamide ready-made gels (NuPAGE Bis-Tris gels, Life technologies, Grand Island, NY, USA) and then transferred onto PVDF membranes. Blots were blocked with $10 \%$ non-fat dried milk for $1 \mathrm{~h}$ and then incubated overnight with specific primary antibodies at $4{ }^{\circ} \mathrm{C}$ on a rotating shaker. Membranes were washed thrice with PBST and incubated with corresponding HRP-conjugated secondary antibody for $90 \mathrm{~min}$. Proteins were then visualized using ECL detection reagents
(SuperSignal West Femto Maximum Sensitivity Substrate; Life technologies) using LI-COR Odyssey Fc image system.

Total ER isolation. Total ER fractions were isolated from GTM cells using ER enrichment kit (cat \# NBP2-29482, Novus Biologicals, LLC). GTM3 cells were grown on $10 \mathrm{~cm}$ dishes and treated with plasmids expressing ATF4. Cells were lysed using $2 \mathrm{ml}$ isosmotic homogenization buffer containing protease inhibitor cocktail. Lysates were centrifuged at $1000 \times g$ for $10 \mathrm{~min}$ at $4{ }^{\circ} \mathrm{C}$. The pellet containing nuclei and cell debris was discarded and supernatant was centrifuged at $12,000 \times g$ for $15 \mathrm{~min}$ at $4^{\circ} \mathrm{C}$. The pellet (mitochondria and cell debris) was discarded and supernatant was ultra-centrifuged at 90,000 $\times g$ (Beckman ultracentrifuge) for $60 \mathrm{~min}$ at $4{ }^{\circ} \mathrm{C}$. The supernatant was discarded and pellet containing the total ER fraction was resuspended in $1 \times$ suspension buffer containing protease inhibitor cocktail and was subjected to Western blot analysis.

Surface sensing of translation (SUnSET) assay. The SUnSET assay was carried out as described previously ${ }^{37,91}$. Primary human TM cells from normal and glaucoma donor eyes or GTM3 cells were plated in $10 \mathrm{~cm}$ tissue culture dishes and treated with vehicle (0.1\% ethanol), Dex $(100 \mathrm{nM})$, and Dex plus Ad5.ATF4 $\Delta$ RK for $36 \mathrm{~h}$ or transfected with plasmids expressing ATF 4 or CHOP for $48 \mathrm{~h}$. TM cells were then incubated with puromycin $(10 \mu \mathrm{g} / \mathrm{ml})$ for $30 \mathrm{~min}$ before harvesting cell lysates. Cells were washed thrice with $1 \times$ PBS and used for the isolation of total ER fractions. To quantify the amount of puromycin incorporated in the newly synthesized proteins in the ER, the ER lysates were subjected to Western blot analysis using mouse anti-puromycin primary antibody (1:10,000 dilution) and goat anti rabbit-HRP secondary antibody. GAPDH was used as a loading control.

Reporting summary. Further information on research design is available in the Nature Research Reporting Summary linked to this article.

\section{Data availability}

All data generated and analyzed during this study are included in this published article (and its supplementary information files). Source data are provided with this paper.

Received: 16 April 2019; Accepted: 8 October 2020;

Published online: 05 November 2020

\section{References}

1. Allingham, R. R., Liu, Y. \& Rhee, D. J. The genetics of primary open-angle glaucoma: a review. Exp. Eye Res. 88, 837-844 (2009).

2. Kwon, Y. H., Fingert, J. H., Kuehn, M. H. \& Alward, W. L. Primary open-angle glaucoma. New Engl. J. Med. 360, 1113-1124 (2009).

3. Quigley, H. A. Neuronal death in glaucoma. Prog. Retin. Eye Res. 18, 39-57 (1999).

4. Quigley, H. A. \& Broman, A. T. The number of people with glaucoma worldwide in 2010 and 2020. Br. J. Ophthalmol. 90, 262-267 (2006).

5. Quigley, H. A. et al. The prevalence of glaucoma in a population-based study of Hispanic subjects: Proyecto VER. Arch. Ophthalmol. 119, 1819-1826 (2001).

6. Rodriguez, J. et al. Causes of blindness and visual impairment in a populationbased sample of U.S. Hispanics. Ophthalmology 109, 737-743 (2002).

7. Budenz, D. L. et al. Blindness and visual impairment in an urban West African population: the Tema Eye Survey. Ophthalmology 119, 1744-1753 (2012).

8. Budenz, D. L. et al. Prevalence of glaucoma in an urban West African population: the Tema Eye Survey. JAMA Ophthalmol. 131, 651-658 (2013).

9. Kapetanakis, V. V. et al. Global variations and time trends in the prevalence of primary open angle glaucoma (POAG): a systematic review and meta-analysis. Br. J. Ophthalmol. 100, 86-93 (2016).

10. Rosenthal, J. \& Leske, M. C. Open-angle glaucoma risk factors applied to clinical area. J. Am. Optom. Assoc. 51, 1017-1024 (1980).

11. Rohen, J. W., Lutjen-Drecoll, E., Flugel, C., Meyer, M. \& Grierson, I. Ultrastructure of the trabecular meshwork in untreated cases of primary openangle glaucoma (POAG). Exp. Eye Res. 56, 683-692 (1993).

12. Bill, A. Editorial: the drainage of aqueous humor. Investig. Ophthalmol. 14, 1-3 (1975).

13. Acott, T. S. \& Kelley, M. J. Extracellular matrix in the trabecular meshwork. Exp. Eye Res. 86, 543-561 (2008).

14. Zhang, X., Ognibene, C. M., Clark, A. F. \& Yorio, T. Dexamethasone inhibition of trabecular meshwork cell phagocytosis and its modulation by glucocorticoid receptor beta. Exp. Eye Res. 84, 275-284 (2007).

15. Alvarado, J., Murphy, C. \& Juster, R. Trabecular meshwork cellularity in primary open-angle glaucoma and nonglaucomatous normals. Ophthalmology 91, 564-579 (1984). 
16. Vranka, J. A., Kelley, M. J., Acott, T. S. \& Keller, K. E. Extracellular matrix in the trabecular meshwork: intraocular pressure regulation and dysregulation in glaucoma. Exp. Eye Res. 133, 112-125 (2015).

17. Clark, A. F., Miggans, S. T., Wilson, K., Browder, S. \& McCartney, M. D. Cytoskeletal changes in cultured human glaucoma trabecular meshwork cells. J. Glaucoma 4, 183-188 (1995).

18. Hoare, M. J. et al. Cross-linked actin networks (CLANs) in the trabecular meshwork of the normal and glaucomatous human eye in situ. Investig. Ophthalmol. Vis. Sci. 50, 1255-1263 (2009).

19. Matsumoto, Y. \& Johnson, D. H. Trabecular meshwork phagocytosis in glaucomatous eyes. Ophthalmologica 211, 147-152 (1997).

20. Resch, Z. T. \& Fautsch, M. P. Glaucoma-associated myocilin: a better understanding but much more to learn. Exp. Eye Res. 88, 704-712 (2009).

21. Wordinger, R. J. \& Clark, A. F. Effects of glucocorticoids on the trabecular meshwork: towards a better understanding of glaucoma. Prog. Retin. Eye Res. 18, 629-667 (1999)

22. Fingert, J. H., Stone, E. M., Sheffield, V. C. \& Alward, W. L. Myocilin glaucoma. Surv. Ophthalmol. 47, 547-561 (2002).

23. Stone, E. M. et al. Identification of a gene that causes primary open angle glaucoma. Science 275, 668-670 (1997).

24. Clark, A. F. \& Wordinger, R. J. The role of steroids in outflow resistance. Exp. Eye Res. 88, 752-759 (2009).

25. Armaly, M. F. \& Becker, B. Intraocular pressure response to topical corticosteroids. Fed. Proc. 24, 1274-1278 (1965).

26. Becker, B. \& Mills, D. W. Corticosteroids and Intraocular Pressure. Arch. Ophthalmol. 70, 500-507 (1963).

27. Bernstein, H. N., Mills, D. W. \& Becker, B. Steroid-induced elevation of intraocular pressure. Arch. Ophthalmol. 70, 15-18 (1963).

28. Kersey, J. P. \& Broadway, D. C. Corticosteroid-induced glaucoma: a review of the literature. Eye 20, 407-416 (2006).

29. Becker, B. Intraocular pressure response to topical corticosteroids. Investig. Ophthalmol. 4, 198-205 (1965).

30. Armaly, M. F. Effect of corticosteroids on intraocular pressure and fluid dynamics. Ii. The effect of dexamethasone in the glaucomatous eye. Arch. Ophthalmol. 70, 492-499 (1963).

31. Steely, H. T. et al. The effects of dexamethasone on fibronectin expression in cultured human trabecular meshwork cells. Investig. Ophthalmol. Vis. Sci. 33, 2242-2250 (1992).

32. Dickerson, J. E. Jr, Steely, H. T. Jr, English-Wright, S. L. \& Clark, A. F. The effect of dexamethasone on integrin and laminin expression in cultured human trabecular meshwork cells. Exp. Eye Res. 66, 731-738 (1998).

33. Zhou, L., Li, Y. \& Yue, B. Y. Glucocorticoid effects on extracellular matrix proteins and integrins in bovine trabecular meshwork cells in relation to glaucoma. Int. J. Mol. Med. 1, 339-346 (1998).

34. Yun, A. J., Murphy, C. G., Polansky, J. R., Newsome, D. A. \& Alvarado, J. A. Proteins secreted by human trabecular cells. Glucocorticoid and other effects. Investig. Ophthalmol. Vis. Sci. 30, 2012-2022 (1989).

35. Kroeger, H., Chiang, W. C., Felden, J., Nguyen, A. \& Lin, J. H. ER stress and unfolded protein response in ocular health and disease. FEBS J. 286, 399-412 (2019).

36. Peters, J. C., Bhattacharya, S., Clark, A. F. \& Zode, G. S. Increased endoplasmic reticulum stress in human glaucomatous trabecular meshwork cells and tissues. Investig. Ophthalmol. Vis. Sci. 56, 3860-3868 (2015).

37. Kasetti, R. B., Maddineni, P., Millar, J. C., Clark, A. F. \& Zode, G. S. Increased synthesis and deposition of extracellular matrix proteins leads to endoplasmic reticulum stress in the trabecular meshwork. Sci. Rep. 7, 14951 (2017).

38. Zode, G. S. et al. Reduction of ER stress via a chemical chaperone prevents disease phenotypes in a mouse model of primary open angle glaucoma. J. Clin. Investig. 121, 3542-3553 (2011).

39. Beckel, J. M. et al. Mechanosensitive release of adenosine $5^{\prime}$-triphosphate through pannexin channels and mechanosensitive upregulation of pannexin channels in optic nerve head astrocytes: a mechanism for purinergic involvement in chronic strain. Glia 62, 1486-1501 (2014).

40. Anholt, R. R. \& Carbone, M. A. A molecular mechanism for glaucoma: endoplasmic reticulum stress and the unfolded protein response. Trends Mol. Med. 19, 586-593 (2013)

41. Jain, A. et al. CRISPR-Cas9-based treatment of myocilin-associated glaucoma. Proc. Natl Acad. Sci. USA 114, 11199-11204 (2017).

42. Schroder, M. \& Kaufman, R. J. The mammalian unfolded protein response. Annu. Rev. Biochem. 74, 739-789 (2005).

43. Yoshida, H. ER stress and diseases. FEBS J. 274, 630-658 (2007).

44. Tabas, I. \& Ron, D. Integrating the mechanisms of apoptosis induced by endoplasmic reticulum stress. Nat. Cell Biol. 13, 184-190 (2011).

45. Sano, R. \& Reed, J. C. ER stress-induced cell death mechanisms. Biochim. Biophys. Acta 1833, 3460-3470 (2013).

46. Ron, D. \& Walter, P. Signal integration in the endoplasmic reticulum unfolded protein response. Nat. Rev. Mol. Cell Biol. 8, 519-529 (2007).
47. Brush, M. H., Weiser, D. C. \& Shenolikar, S. Growth arrest and DNA damageinducible protein GADD34 targets protein phosphatase 1 alpha to the endoplasmic reticulum and promotes dephosphorylation of the alpha subunit of eukaryotic translation initiation factor 2. Mol. Cell. Biol. 23, 1292-1303 (2003).

48. Rutkowski, D. T. et al. Adaptation to ER stress is mediated by differential stabilities of pro-survival and pro-apoptotic mRNAs and proteins. PLoS Biol. 4, e374 (2006).

49. Lin, J. H., Li, H., Zhang, Y., Ron, D. \& Walter, P. Divergent effects of PERK and IRE1 signaling on cell viability. PLoS ONE 4, e4170 (2009).

50. Walter, F., Schmid, J., Dussmann, H., Concannon, C. G. \& Prehn, J. H. Imaging of single cell responses to ER stress indicates that the relative dynamics of IRE1/XBP1 and PERK/ATF4 signalling rather than a switch between signalling branches determine cell survival. Cell Death Differ. 22, 1502-1516 (2015)

51. Han, J. et al. ER-stress-induced transcriptional regulation increases protein synthesis leading to cell death. Nat. Cell Biol. 15, 481-490 (2013).

52. Iurlaro, R. \& Munoz-Pinedo, C. Cell death induced by endoplasmic reticulum stress. FEBS J. 283, 2640-2652 (2016).

53. Patel, G. C. et al. Dexamethasone-induced ocular hypertension in mice: effects of myocilin and route of administration. Am. J. Pathol. 187, 713-723 (2017)

54. Millar, J. C., Clark, A. F. \& Pang, I. H. Assessment of aqueous humor dynamics in the mouse by a novel method of constant-flow infusion. Investig. Ophthalmol. Vis. Sci. 52, 685-694 (2011).

55. Millar, J. C., Phan, T. N., Pang, I. H. \& Clark, A. F. Strain and age effects on aqueous humor dynamics in the mouse. Investig. Ophthalmol. Vis. Sci. 56, 5764-5776 (2015)

56. Challa, P. et al. Lentiviral mediated gene delivery to the anterior chamber of rodent eyes. Mol. Vis. 11, 425-430 (2005).

57. Loewen, N. et al. Genetic modification of human trabecular meshwork with lentiviral vectors. Hum. Gene Ther. 12, 2109-2119 (2001).

58. Pang, I. H., Millar, J. C. \& Clark, A. F. Elevation of intraocular pressure in rodents using viral vectors targeting the trabecular meshwork. Exp. Eye Res. 141, 33-41 (2015)

59. Chou, T. H., Bohorquez, J., Toft-Nielsen, J., Ozdamar, O. \& Porciatti, V. Robust mouse pattern electroretinograms derived simultaneously from each eye using a common snout electrode. Investig. Ophthalmol. Vis. Sci. 55, 2469-2475 (2014)

60. Nashine, S., Liu, Y., Kim, B. J., Clark, A. F. \& Pang, I. H. Role of C/EBP homologous protein in retinal ganglion cell death after ischemia/reperfusion injury. Investig. Ophthalmol. Vis. Sci. 56, 221-231 (2014).

61. Karali, A., Russell, P., Stefani, F. H. \& Tamm, E. R. Localization of myocilin/ trabecular meshwork-inducible glucocorticoid response protein in the human eye. Investig. Ophthalmol. Vis. Sci. 41, 729-740 (2000).

62. Takahashi, H. et al. Mouse myocilin (Myoc) gene expression in ocular tissues Biochem. Biophys. Res. Commun. 248, 104-109 (1998).

63. Tamm, E. R., Russell, P., Epstein, D. L., Johnson, D. H. \& Piatigorsky, J. Modulation of myocilin/TIGR expression in human trabecular meshwork. Investig. Ophthalmol. Vis. Sci. 40, 2577-2582 (1999).

64. Kasetti, R. B., Phan, T. N., Millar, J. C. \& Zode, G. S. Expression of mutant myocilin induces abnormal intracellular accumulation of selected extracellular matrix proteins in the trabecular meshwork. IOVS 57, 6058-6069 (2016).

65. Lu, M. et al. Opposing unfolded-protein-response signals converge on death receptor 5 to control apoptosis. Science 345, 98-101 (2014).

66. Lange, P. S. et al. ATF4 is an oxidative stress-inducible, prodeath transcription factor in neurons in vitro and in vivo. J. Exp. Med. 205, 1227-1242 (2008)

67. Wang, C. et al. ATF4 deficiency protects hepatocytes from oxidative stress via inhibiting CYP2E1 expression. J. Cell. Mol. Med. 18, 80-90 (2014).

68. Quiros, P. M. et al. Multi-omics analysis identifies ATF4 as a key regulator of the mitochondrial stress response in mammals. J. Cell Biol. 216, 2027-2045 (2017).

69. Vahabikashi, A. et al. Increased stiffness and flow resistance of the inner wall of Schlemm's canal in glaucomatous human eyes. Proc. Natl Acad. Sci. USA https://doi.org/10.1073/pnas.1911837116 (2019).

70. Overby, D. R. et al. Altered mechanobiology of Schlemm's canal endothelial cells in glaucoma. Proc. Natl Acad. Sci. USA 111, 13876-13881 (2014).

71. Last, J. A. et al. Elastic modulus determination of normal and glaucomatous human trabecular meshwork. Investig. Ophthalmol. Vis. Sci. 52, 2147-2152 (2011).

72. Wang, K. et al. Estimating human trabecular meshwork stiffness by numerical modeling and advanced OCT imaging. Investig. Ophthalmol. Vis. Sci. 58, 4809-4817 (2017)

73. Sidrauski, C., McGeachy, A. M., Ingolia, N. T. \& Walter, P. The small molecule ISRIB reverses the effects of eIF2alpha phosphorylation on translation and stress granule assembly. eLife 4, https://doi.org/10.7554/ eLife.05033 (2015). 
74. Rabouw, H. H. et al. Small molecule ISRIB suppresses the integrated stress response within a defined window of activation. Proc. Natl Acad. Sci. USA 116, 2097-2102 (2019).

75. Zyryanova, A. F. et al. Binding of ISRIB reveals a regulatory site in the nucleotide exchange factor eIF2B. Science 359, 1533-1536 (2018).

76. Kasetti, R. B., Phan, T. N., Millar, J. C. \& Zode, G. S. Expression of mutant myocilin induces abnormal intracellular accumulation of selected extracellular matrix proteins in the trabecular meshwork. Investig. Ophthalmol. Vis. Sci. 57, 6058-6069 (2016).

77. Doyle, K. M. et al. Unfolded proteins and endoplasmic reticulum stress in neurodegenerative disorders. J. Cell. Mol. Med. 15, 2025-2039 (2011).

78. Hoozemans, J. J., van Haastert, E. S., Nijholt, D. A., Rozemuller, A. J. \& Scheper, W. Activation of the unfolded protein response is an early event in Alzheimer's and Parkinson's disease. Neurodegener. Dis. 10, 212-215 (2012).

79. Soto, C. \& Estrada, L. D. Protein misfolding and neurodegeneration. Arch. Neurol. 65, 184-189 (2008).

80. Pakos-Zebrucka, K. et al. The integrated stress response. EMBO Rep. 17, 1374-1395 (2016).

81. Shepard, A. R. et al. Adenoviral gene transfer of active human transforming growth factor-\{beta\}2 elevates intraocular pressure and reduces outflow facility in rodent eyes. Investig. Ophthalmol. Vis. Sci. $\mathbf{5 1}$ 2067-2076 (2010).

82. Yang, Y. et al. Transcription factor C/EBP homologous protein in health and diseases. Front. Immunol. 8, 1612 (2017).

83. Su, N. \& Kilberg, M. S. C/EBP homology protein (CHOP) interacts with activating transcription factor 4 (ATF4) and negatively regulates the stressdependent induction of the asparagine synthetase gene. J. Biol. Chem. 283, 35106-35117 (2008).

84. Marciniak, S. J. et al. CHOP induces death by promoting protein synthesis and oxidation in the stressed endoplasmic reticulum. Genes Dev. 18, 3066-3077 (2004).

85. Wong, Y. L. et al. The small molecule ISRIB rescues the stability and activity of vanishing white matter disease eIF2B mutant complexes. eLife 7, https://doi. org/10.7554/eLife.32733 (2018)

86. Tsai, J. C. et al. Structure of the nucleotide exchange factor eIF2B reveals mechanism of memory-enhancing molecule. Science 359, https://doi.org/ 10.1126/science.aaq0939 (2018).

87. Wang, Y. et al. Endoplasmic reticulum stress response of trabecular meshwork stem cells and trabecular meshwork cells and protective effects of activated PERK pathway. Investig. Ophthalmol. Vis. Sci. 60, 265-273 (2019).

88. Zode, G. S. et al. Ocular-specific ER stress reduction rescues glaucoma in murine glucocorticoid-induced glaucoma. J. Clin. Investig. 124, 1956-1965 (2014).

89. Wang, W. H., Millar, J. C., Pang, I. H., Wax, M. B. \& Clark, A. F. Noninvasive measurement of rodent intraocular pressure with a rebound tonometer. Investig. Ophthalmol. Vis. Sci. 46, 4617-4621 (2005).

90. Kasetti, R. B., Maddineni, P., Millar, J. C., Clark, A. F. \& Zode, G. S. Increased synthesis and deposition of extracellular matrix proteins leads to endoplasmic reticulum stress in the trabecular meshwork. Sci. Rep.-UK 7, 14951 (2017).
91. Schmidt, E. K., Clavarino, G., Ceppi, M. \& Pierre, P. SUnSET, a nonradioactive method to monitor protein synthesis. Nat. Methods 6, 275-277 (2009).

\section{Acknowledgements}

The authors would like to thank Dr. Abbot Clark, Dr. Colleen McDowell, Sherri Harris, and Sandra Maansson for assistance with some experiments. These studies were supported by the National Institutes of Health (EY028616 and EY026177) and bright focus glaucoma foundation.

\section{Author contributions}

R.B.K. and G.S.Z. designed research studies, analyzed data, provided reagents and wrote the manuscript. P.M., P.D.P., C.C.S., J.C.M. V.R. and V.C.S. assisted in conducting key experiments, provided reagents, and assisted in manuscript preparation. All authors discussed the results and implications and commented on the manuscript at all stages.

\section{Competing interests}

The authors declare no competing interests.

\section{Additional information}

Supplementary information is available for this paper at https://doi.org/10.1038/s41467020-19352-1.

Correspondence and requests for materials should be addressed to G.S.Z.

Peer review information Nature Communications thanks the anonymous reviewer(s) for their contribution to the peer review of this work. Peer reviewer reports are available.

Reprints and permission information is available at http://www.nature.com/reprints

Publisher's note Springer Nature remains neutral with regard to jurisdictional claims in published maps and institutional affiliations.

pen Access This article is licensed under a Creative Commons Attribution 4.0 International License, which permits use, sharing, adaptation, distribution and reproduction in any medium or format, as long as you give appropriate credit to the original author(s) and the source, provide a link to the Creative Commons license, and indicate if changes were made. The images or other third party material in this article are included in the article's Creative Commons license, unless indicated otherwise in a credit line to the material. If material is not included in the article's Creative Commons license and your intended use is not permitted by statutory regulation or exceeds the permitted use, you will need to obtain permission directly from the copyright holder. To view a copy of this license, visit http://creativecommons.org/ licenses/by/4.0/.

(C) The Author(s) 2020 